\title{
Social inequalities in alcohol-related adult mortality by National Statistics Socio-economic Classification, England and Wales, 2001-03
}

Veronique Siegler, Alaa Al-Hamad, Brian Johnson, Claudia Wells Office for National Statistics and Nick Sheron Southampton University

\section{Abstract \\ Background}

This article is the first analysis of the social inequalities in adult alcohol-related mortality in England and Wales at the start of the 21st century, using the National Statistics Socioeconomic Classification (NS-SEC). It presents the socio-economic patterns of alcoholrelated mortality by gender, age and region, for England and Wales as a whole, Wales and the regions of England.

\section{Methods}

Death registrations provided the number of deaths for working age adults, using the National Statistics definition of alcohol-related mortality. Population estimates for England and Wales in 2001-03 were used to estimate alcohol-related mortality rates by sex, five-year age group, NS-SEC and region. Inequalities were measured using ratios of alcohol-related mortality rates between the least and most advantaged classes.

\section{Results}

There were substantial socio-economic variations in adult alcohol-related mortality, with the inequalities being greater for women than for men. The mortality rate of men in the Routine class was 3.5 times those of men in Higher and Managerial occupations, while for women the corresponding figure was $\mathbf{5 . 7}$ times. Greater socio-economic inequalities in mortality were observed for men aged 25-49 than for men aged 50-64; however the highest mortality rate of men occurred for Routine workers aged 50-54. Women in the Routine class experienced mortality rates markedly higher than other classes. The highest mortality rate of women also occurred for Routine workers, but at a younger age than for men (45-49). Within England, the North-West showed the largest inequalities, with particularly high rates in the Routine class for both sexes. In general, there was no association between levels of mortality and socio-economic gradients in mortality across the English regions and Wales. 


\section{Conclusions}

Rates of alcohol-related mortality in England and Wales increased significantly for people between the early 1990 s and early 21 st century, and were substantially greater for those in more disadvantaged socio-economic classes. There is also evidence that these socioeconomic differences were greater at younger ages, especially for men at ages 25-49. 


\section{Contents}

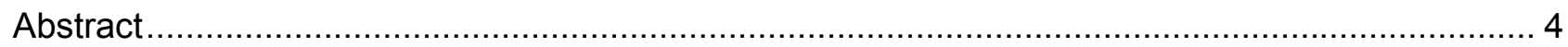

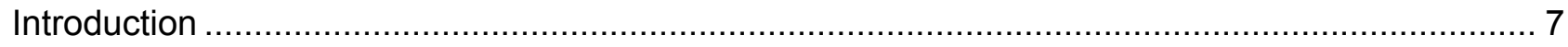

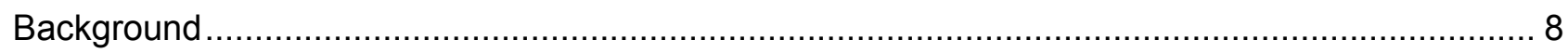

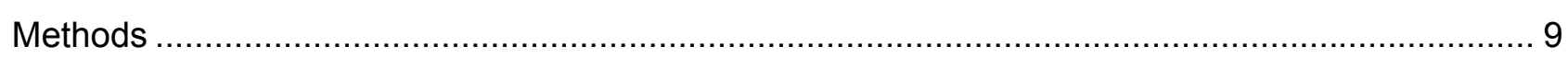

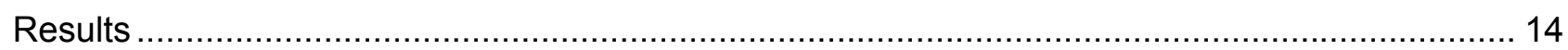

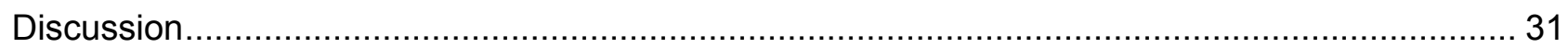

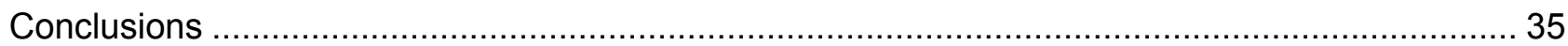

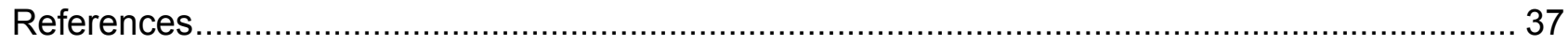

\section{List of Figures}

Figure 1 Age-specific mortality rates from alcohol-related causes of death by five-year age group and NS-SEC, men aged 25-64, England and Wales, 2001-03 .................. 17

Figure 2 Age-specific mortality rates from alcohol-related causes of death by five-year age group and NS-SEC, women aged 25-59, England and Wales, 2001-03

Figure 3 Age-standardised mortality rates from alcohol-related causes of death by NS-SEC, men aged 25-64 and women aged 25-59, England and Wales, 2001-03 22

Figure 4 Age-standardised mortality rates from alcohol-related causes of death by NS-SEC, men aged 25-64 and women aged 25-59, England and Wales, the regions of England and Wales, 2001-03.

Figure 5 Socio-economic gradients in mortality for alcohol-related causes of death, for men aged 25-64, in England and Wales, the regions of England and Wales, 2001-03. 28

Figure 6 Socio-economic gradients in mortality for alcohol-related causes of death, for women aged 25-59, in England and Wales, the regions of England and Wales, 2001-03 


\section{List of Tables}

Table 1 Optimised population estimates (person years at risk) by NS-SEC classification and by region, men aged 25-64, 2001-03 ........................................................ 13

Table 2 Optimised population estimates (person years at risk) by NS-SEC classification and by region, women aged 25-59, 2001-03

Table $3 \quad$ Number of deaths attributed to specific alcohol-related causes, men aged 25-64 and women aged 25-59, England and Wales, 2001-03

Table 4 Adjusted number of alcohol-related deaths by NS-SEC classification and by region, men aged 25-64, 2001-03.

Table 5 Adjusted number of alcohol-related deaths by NS-SEC and by region, women aged

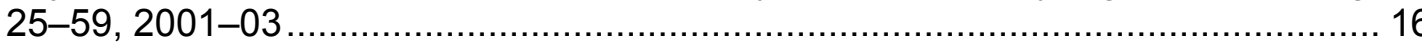

Table 6 Age-specific mortality rates by five year age group and NS-SEC classification, men

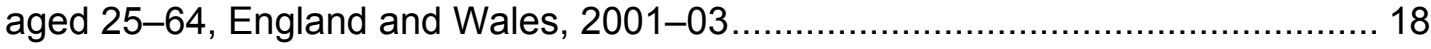

Table 7 Age-specific mortality rates by five year age group and NS-SEC classification, women aged 25-59, England and Wales, 2001-03

Table $8 \quad$ Age-standardised mortality rates from alcohol-related causes of death, by NS-SEC classification and region, men aged 25-64, England and Wales, 2001-03

Table 9 Age-standardised mortality rates from alcohol-related causes of death, by NS-SEC classification and region, women aged 25-59, England and Wales, 2001-03.

\section{Introduction}

Excessive consumption of alcohol is a major preventable cause of premature mortality, disability and injury. Rates of alcohol-related mortality in the United Kingdom (UK) have increased significantly since the early 1990s. The number of alcohol-related deaths in England and Wales doubled between 1991 and 2008, rising from 3,415 (6.4 per 100,000 population) in 1991 to 7,344 (12.4 per 100,000) in 2008 (Office for National Statistics (ONS), 2011). Eighty-five per cent of alcohol-related deaths in this period were attributed to chronic liver diseases and 65 per cent of deaths occurred in men. The most recent data indicated that there were 7,099 alcohol-related deaths in England and Wales in 2009 (11.8 per 100,000), representing a 3.3 per cent drop in deaths compared to 2008 (ONS, 2011).

In England and Wales, socio-economic class and deprivation have been identified as risk factors for alcohol-related mortality and large geographic variations have also been observed (Breakwell et al., 2007; Harrison and Gardiner, 1999).

This article describes patterns of social inequalities in alcohol-related mortality for men and women of working age, in England and Wales using the National Statistics Socio-economic Classification (NS-SEC). The study was restricted to the period 2001 to 2003 as a consequence of constraints on 
the availability of population data suitably classified by NS-SEC. The article also reports on age and geographic variations in inequalities in alcohol-related mortality for both men and women.

\section{Background}

The high impact of alcohol misuse on ill-health, intentional and non-intentional injuries, and mortality in England and Wales has long been reported in the literature (WHO, 2011; Sheron et al., 2011; House of Commons Health Committee, 2010; Baker and Rooney, 2003). Binge drinking and long-term heavy drinking are both known to be associated with harm to physical and psychological health, and can also be a cause of harm to others through road traffic accidents, violence and crime (House of Commons Health Committee, 2010). The Department of Health estimated that 7 per cent of all hospital admissions are alcohol-related, costing the NHS £2.7 billion a year (Department of Health, 2010).

Drinking patterns in the UK have changed in the second half of the 20th century, both in terms of the volume of alcohol consumed and the demographic profile of those drinking. The average consumption of alcohol per head in the UK increased from three-and-a-half litres of pure alcohol per head in 1947 to nine-and-a-half litres in 2004/05 and fell to 8.9 litres in 2008 (BBPA and HM Revenue and Customs, 2009). General Lifestyle Survey (GLF) data for 2009 suggested that 7 per cent of men in Great Britain were drinking at levels defined by the Department of Health as 'higher risk' (more than 50 units per week) and a further 19 per cent were drinking at levels defined as 'increasing risk' (between 21 and 50 units per week) (GLF, 2009).

The biggest demographic changes in drinking patterns in recent years have been the increases among women and young people. The GLF, formerly known as the General Household Survey (GHS) recorded a marked rise in overall weekly alcohol consumption among women between 1988 and 2002, in particular among young women aged 16-24. In 1988, 15 per cent of women in this age group were drinking more than 14 units per week, whereas in 2002 it was 33 per cent. However, there was a fall in the proportion of women aged 16-24 drinking more than 14 units per week to 19 per cent in 2006. A new methodology of converting volumes of alcoholic drinks to units of alcohol was introduced in 2006. The figures using this new methodology indicated that 24 per cent of women aged 16-24 were exceeding 14 units per week in 2006 and 23 per cent in 2009.

The mean alcohol consumption by children aged 11-15 in England who had drunk alcohol in the preceding week doubled between 1990 and 2006, according to a survey by the NHS Health and Social Care Information Centre (2010). The new methodology of converting volumes of alcoholic drinks to units of alcohol showed that the mean alcohol consumption of children aged 11-15 in England who had drunk alcohol in the week preceding the survey was 12.7 units in 2007 , increased to 14.6 units in 2008 and reduced to 11.6 units in 2009.

Thus, while evidence on the most recent trends is equivocal, there is no question that alcohol consumption in England and Wales has increased substantially over the last twenty years for both sexes and all ages.

Social class, occupation and deprivation have been identified as factors associated with alcoholrelated mortality in several studies (Erskine et al., 2010; Breakwell et al., 2007; Romeri et al., 2007; Harrison and Gardiner, 1999; Makela et al., 1997). Makela et al. (1997) found that alcohol-related 
mortality in Finland was almost three times higher for men and twice as high for women when comparing manual workers with senior non-manual employees. The work of Harrison and Gardiner (1999) highlighted higher mortality rates for men in manual classes compared with professional social classes in Great Britain in 1988-94, with a greater difference at younger ages.

ONS reported on alcohol-related mortality by occupation in England and Wales between 2001 and 2005 (Romeri et al., 2007). It was shown that the occupational groups with the lowest alcoholrelated mortality were Managers, Senior Officials and Professionals. Erskine et al. (2010) provided further evidence of association between alcohol-related mortality and socio-economic deprivation in England and Wales between 1999 and 2003, with the greatest inequalities observed among people aged $25-44$ years.

The Marmot Review (2010) mentioned an association between alcohol-related hospital admissions and high levels of deprivation for both men and women, with particularly high rates of admission for those areas among the most deprived quintile of England and Wales. Binge drinking was also reported to be most common among those living in deprived areas.

This article aims to contribute to the debate on socio-economic inequalities in alcohol-related deaths by assessing the patterns of age-specific mortality rates for men and women of working age and by examining the gender and regional socio-economic variations in age-standardised mortality rates. The analysis of these rates is presented for the first time by the National Statistics Socioeconomic Classification (NS-SEC) whereas previous socio-economic alcohol-related mortality analysis used the Registrar General's Social Class (RGSC). The analysis will cover England and Wales as a whole and it will also investigate variations in socio-economic inequalities across Wales and the regions of England. England as a whole on the other hand will not be covered by this analysis due to the close proximity of its results to those of England and Wales. However data on rates for England can be found in separate web tables on the ONS website.

www.statistics.gov.uk/hsq/downloads/hsq50art1.xls

\section{Methods}

In this section the measure of socio-economic class used along with the definitions of the alcoholrelated deaths and regions are described in more detail. The data sources used in the analysis and the outcome measures adopted in this article are also described.

\section{The National Statistics Socio-economic Classification (NS-SEC)}

The National Statistics Socio-economic Classification (NS-SEC) was developed with the aim of replacing both the Registrar General's Social Class (RGSC) and the Socio-economic Groups (SEG) in official statistics, due to its improved conceptual and theoretical basis (Rose and Pevalin et al., 2003). The conceptual basis for the NS-SEC is the structure of employment relations operating in modern developed economies. Occupations are differentiated in terms of reward mechanisms, promotion prospects, autonomy and job security. The most advantaged NS-SEC classes (Higher managerial and professional) typically exhibit personalised reward structures, have good opportunities for advancement, relatively high levels of autonomy within the job, and are relatively secure. These attributes tend to be reversed for the most disadvantaged class (Routine). 
The operational categories of the NS-SEC can be derived by three principal methods, full, reduced or simplified depending on the data available. The full NS-SEC requires occupational information coded to the Standard Occupational Classification 2000 (SOC 2000), details of employment status and the size of the organisation. The reduced NS-SEC requires only the SOC 2000 occupational classification and details of employment status (Simplified NS-SEC requires occupation only). For the purpose of this article, since the size of the organisation is not collected at death registration, the reduced method was used to derive NS-SEC.

For analysis purposes NS-SEC is nested so that the operational categories can offer maximum flexibility in terms of the different collapses possible (Rose and Pevalin, 2003). Box 1 presents the eight-class version of the NS-SEC that was used in this analysis.

For the purpose of this article, those who had never worked, the long-term unemployed, full-time students, those with an occupation inadequately described or unclassified for other reasons are referred to as the 'non-occupied group' and are excluded from the analysis, being composed of a disparate range of people.

\section{Definition of alcohol-related mortality}

The National Statistics definition of alcohol-related deaths based on the International Classification of Diseases, Tenth Revision (ICD10) was used (ONS, 2006). It includes only those causes regarded as being most directly due to alcohol consumption (Box 2). It does not include other diseases where alcohol has been shown to have some causal relationship, such as cancers of the mouth, oesophagus and liver. The definition includes all deaths from chronic liver disease and cirrhosis (excluding biliary cirrhosis), even when alcohol is not specifically mentioned on the death certificate. Apart from deaths due to poisoning with alcohol (accidental, intentional or undetermined), this definition excludes any other external causes of death, such as road traffic and other accidents. The definition allows for consistent comparisons over time for those deaths most clearly associated with alcohol consumption. 


\section{Box 1 National Statistics Socio-economic Classification Analytic classes}

Analytic classes

Eight class version

Higher managerial

1 and professional occupations

2

3

4

5

6

7

Non-occupied

\section{Examples of occupations include:}

Large employers

1.1 and higher

managerial

Higher

1.2 professional

Lower managerial and professional

Intermediate

Small employers and own account workers

Lower supervisory and technical

Semi-routine

Routine 


\begin{tabular}{|ll|}
\hline Box 2 & $\begin{array}{l}\text { The National Statistics definition of alcohol-related deaths } \\
\text { using the International Classification of Diseases, Tenth } \\
\text { Revision (ICD-10) }\end{array}$ \\
\hline ICD-10 Code & Description \\
F10 & Mental and behavioural disorders due to use of alcohol \\
G31.2 & Degeneration of nervous system due to alcohol \\
G62.1 & Alcoholic polyneuropathy \\
I42.6 & Alcoholic cardiomyopathy \\
K29.2 & Alcoholic gastritis \\
K70 & Alcoholic liver disease \\
K73 & Chronic hepatitis, not elsewhere classified \\
K74 & $\begin{array}{l}\text { Fibrosis and cirrhosis of liver (Excluding K74.3-K74.5; Biliary } \\
\text { cirrhosis) }\end{array}$ \\
K86.0 & Alcoholic induced chronic pancreatitis \\
X45 & Accidental poisoning and exposure to alcohol \\
X65 & Intentional self-poisoning by and exposure to alcohol \\
Y15 & Poisoning by and exposure to alcohol, intent undetermined \\
&
\end{tabular}

\section{Data sources for analysis}

\section{Mortality data}

Death registration records were used to count deaths with an underlying cause that were alcoholrelated for working age adults, men aged 25-64 and women aged 25-59, registered in England and Wales in the calendar years 2001 to 2003. These deaths were identified using the International Classification of Diseases, 10th Revision (ICD-10) codes given in Box 2. Information collected at death registration also includes occupational details for both men and women. However, while for men there is good recording of occupational details at death that allows clear allocation of an NS-SEC occupied class, for women this is often not the case. There is a substantial degree of under-reporting of the occupations of women at death which makes it much more difficult to assign them to an NS-SEC occupied class (Langford and Johnson, 2009).

Therefore for women in this analysis, it was thought to be most appropriate to use a 'combined' classification whereby a non-married woman is assigned an NS-SEC class on the basis of her own occupation and employment status and a married women is assigned the most advantaged NSSEC class of either herself or her husband. 
The 'combined' classification has been used previously in an analysis describing social inequalities in adult women mortality (Langford and Johnson, 2009; Erikson, 1984). This combined classification method is not used for men (due to the fact that wives' occupations were rarely recorded).

In the current analysis an adjustment was applied to the mortality data of men and women, to correct for the misallocation of deaths between NS-SEC classes 2 and 3 . This misallocation occurred because some death registration entries for technical workers did not record that the individual concerned had a supervisory role. A further adjustment was applied to female mortality data only. This was to correct for the under-recording of the occupation of women at death. For more detail on the process of adjustment for men see White et al., (2007) and for women see Langford and Johnson, (2009).

\section{Population data}

The population of men and women used in this analysis were based on the adjusted population estimates for England and Wales 2001-03, presented in previous articles on mortality by NS-SEC (Langford and Johnson, 2009; White et al., 2007). For the regional population estimates of men and women in each age group and NS-SEC class, the percentage distributions across Wales and the regions of England were calculated from the 2001 Census data for men and women respectively, as presented in previous articles by Siegler et al., 2008 and Langford et al., 2009. Tables 1 and 2 present the population of men and women of working age in 2001-03, used in this analysis. Breakdown of these populations by five-year age group can be found in separate tables published on the ONS website at: www.statistics.gov.uk/hsq/downloads/hsq50art1.xls

\section{Table $1 \quad$ Optimised population estimates (person years at risk) by NS-SEC classification and by region, men aged 25-64, 2001-03}

\begin{tabular}{|c|c|c|c|c|c|c|c|c|c|c|c|c|}
\hline \multicolumn{2}{|r|}{ NS-SEC analytic classes } & \multirow{2}{*}{$\begin{array}{r}\begin{array}{r}\text { England } \\
\text { and } \\
\text { Wales }\end{array} \\
7,077,427\end{array}$} & \multirow{2}{*}{$\begin{array}{r}\begin{array}{r}\text { North } \\
\text { East }\end{array} \\
224,478\end{array}$} & \multirow{2}{*}{$\begin{array}{r}\begin{array}{r}\text { North } \\
\text { West }\end{array} \\
763,618\end{array}$} & \multirow{2}{*}{$\begin{array}{r}\begin{array}{c}\text { Yorkshire } \\
\text { and The } \\
\text { Humber }\end{array} \\
532,789\end{array}$} & \multirow{2}{*}{$\begin{array}{r}\begin{array}{r}\text { East } \\
\text { Midlands }\end{array} \\
520,728\end{array}$} & \multirow{2}{*}{$\begin{array}{r}\begin{array}{r}\text { West } \\
\text { Midlands }\end{array} \\
631,976\end{array}$} & \multirow{2}{*}{$\begin{array}{r}\begin{array}{r}\text { East of } \\
\text { England }\end{array} \\
822,386\end{array}$} & \multirow{2}{*}{$\begin{array}{c}\text { London } \\
1,231,049\end{array}$} & \multirow{2}{*}{$\begin{array}{r}\begin{array}{r}\text { South } \\
\text { East }\end{array} \\
1,440,310\end{array}$} & \multirow{2}{*}{$\begin{array}{r}\begin{array}{r}\text { South } \\
\text { West }\end{array} \\
636,201\end{array}$} & \multirow{2}{*}{$\begin{array}{c}\text { Wales } \\
273,893\end{array}$} \\
\hline 1 & Higher managerial and professional & & & & & & & & & & & \\
\hline 1.1 & Large employers and higher managerial & $3,176,735$ & 99,248 & 341,644 & 251,394 & 256,874 & 310,860 & 384,813 & 469,235 & 670,931 & 279,373 & 112,363 \\
\hline 1.2 & Higher professional & $3,900,692$ & 125,230 & 421,974 & 281,395 & 263,854 & 321,116 & 437,573 & 761,814 & 769,379 & 356,828 & 161,530 \\
\hline 2 & Lower managerial and professional & $9,212,030$ & 369,126 & $1,050,287$ & 771,128 & 690,722 & 816,892 & $1,046,971$ & $1,504,966$ & $1,632,975$ & 892,132 & 436,831 \\
\hline 3 & Intermediate & $2,438,268$ & 117,072 & 314,897 & 210,893 & 170,158 & 209,400 & 256,245 & 420,647 & 399,767 & 222,538 & 116,650 \\
\hline 4 & Small employers and own account workers & $5,784,013$ & 193,463 & 681,529 & 514,106 & 453,379 & 549,436 & 681,752 & 764,763 & 971,297 & 657,134 & 317,155 \\
\hline & Lower supervisory and technical & $5,660,218$ & 323,010 & 779,204 & 596,767 & 513,919 & 621,863 & 585,381 & 540,472 & 807,444 & 548,852 & 343,307 \\
\hline 6 & Semi-routine & $4,443,052$ & 242,137 & 611,121 & 471,645 & 400,855 & 577,733 & 426,404 & 525,956 & 533,146 & 400,704 & 253,350 \\
\hline \multirow{2}{*}{\multicolumn{2}{|c|}{7 Routine }} & $5,964,793$ & 364,070 & 836,442 & 704,988 & 588,909 & 699,542 & 580,227 & 557,190 & 722,040 & 545,248 & 366,137 \\
\hline & & $40,579,801$ & $1,833,355$ & $5,037,099$ & $3,802,316$ & $3,338,670$ & $4,106,841$ & $4,399,367$ & $5,545,042$ & $6,506,978$ & $3,902,808$ & $2,107,324$ \\
\hline
\end{tabular}




\section{Table $2 \quad$ Optimised population estimates (person years at risk) by NS-SEC classification and by region, women aged 25-59, 2001-03}

\begin{tabular}{|c|c|c|c|c|c|c|c|c|c|c|c|c|}
\hline \multicolumn{2}{|r|}{ NS-SEC analytic classes } & \multirow{2}{*}{$\begin{array}{r}\begin{array}{r}\text { England } \\
\text { and } \\
\text { Wales }\end{array} \\
6,390,536\end{array}$} & \multirow{2}{*}{$\begin{array}{r}\begin{array}{r}\text { North } \\
\text { East }\end{array} \\
212,315\end{array}$} & \multirow{2}{*}{$\begin{array}{r}\begin{array}{r}\text { North } \\
\text { West }\end{array} \\
696,723\end{array}$} & \multirow{2}{*}{$\begin{array}{l}\begin{array}{l}\text { Yorshire } \\
\text { and The } \\
\text { Humber }\end{array} \\
491,871\end{array}$} & \multirow{2}{*}{$\begin{array}{r}\text { East } \\
\text { Midlands }\end{array}$} & \multirow{2}{*}{$\begin{array}{r}\begin{array}{r}\text { West } \\
\text { Midlands }\end{array} \\
565,399\end{array}$} & \multirow{2}{*}{$\begin{array}{r}\begin{array}{r}\text { East of } \\
\text { England }\end{array} \\
739,198\end{array}$} & \multirow{2}{*}{$\begin{array}{c}\text { London } \\
1,084,037\end{array}$} & \multirow{2}{*}{$\begin{array}{r}\begin{array}{r}\text { South } \\
\text { East }\end{array} \\
1,297,885\end{array}$} & \multirow{2}{*}{$\begin{array}{r}\begin{array}{r}\text { South } \\
\text { West }\end{array} \\
572,671\end{array}$} & \multirow{2}{*}{$\begin{array}{r}\text { Wales } \\
254,355\end{array}$} \\
\hline 1 & Higher managerial and professional & & & & & & & & & & & \\
\hline 1.1 & Large employers and higher managerial & $3,193,104$ & 103,045 & 345,913 & 250,131 & 254,146 & 300,555 & 381,798 & 488,042 & 678,768 & 277,013 & 113,692 \\
\hline 1.2 & Higher professional & $3,197,432$ & 109,270 & 350,810 & 241,740 & 221,935 & 264,844 & 357,400 & 595,995 & 619,117 & 295,658 & 140,663 \\
\hline 2 & Lower managerial and professional & $11,308,881$ & 484,781 & $1,360,823$ & 983,221 & 857,396 & $1,041,418$ & $1,233,903$ & $1,777,193$ & $1,893,508$ & $1,095,812$ & 580,825 \\
\hline 3 & Intermediate & $5,458,575$ & 263,048 & 711,937 & 493,906 & 412,682 & 519,860 & 588,004 & 852,497 & 855,800 & 494,247 & 266,595 \\
\hline 4 & Small employers and own account workers & $2,960,311$ & 107,402 & 354,323 & 277,865 & 243,003 & 286,417 & 334,022 & 363,046 & 474,175 & 348,205 & 171,852 \\
\hline 5 & Lower supervisory and technical & $2,890,943$ & 171,857 & 388,966 & 317,292 & 277,102 & 325,265 & 290,747 & 263,382 & 386,434 & 282,575 & 187,324 \\
\hline 6 & Semi-routine & $5,054,560$ & 292,973 & 704,470 & 553,885 & 440,377 & 581,220 & 491,031 & 570,531 & 632,093 & 479,507 & 308,475 \\
\hline \multirow{2}{*}{\multicolumn{2}{|c|}{7 Routine }} & $2,660,774$ & 177,734 & 384,295 & 322,654 & 280,186 & 313,087 & 232,702 & 263,002 & 291,970 & 224,289 & 170,855 \\
\hline & & $36,724,580$ & $1,710,110$ & $4,601,536$ & $3,440,693$ & $2,986,828$ & $3,632,666$ & $3,909,608$ & $5,173,687$ & $5,831,865$ & $3,497,305$ & $1,940,281$ \\
\hline
\end{tabular}

Source: ONS

\section{Outcome measures}

To compare the alcohol-related mortality by NS-SEC analytic classes, for adults of working age, two measures of mortality rates were used. Firstly, age-specific mortality rates by NS-SEC and five-year age groups were calculated for men aged 25-64 and women aged 25-59 in England and Wales, 2001-03. Secondly, mortality rates for men aged 25-64 and women aged 25-59, standardised to the European Standard Population, were calculated for England and Wales as a whole, and by the English regions and Wales, for the period 2001-03. Age-standardised rates are a summary measure allowing populations with differing age structure to be reliably compared.

The ratios of the above mortality rates of the least advantaged to the most advantaged NS-SEC class were also calculated. These ratios are referred to in the article as socio-economic gradients. Confidence intervals were used to assess the significance of these mortality rate ratios between the least and most advantaged classes.

\section{Results}

The total numbers of alcohol related deaths for men and women attributed to specific causes is shown in Table 3. There were 8,938 alcohol-related deaths to men aged 25-64 and 3,613 to women aged 25-59 in England and Wales, during 2001-03. Alcoholic liver disease represented about two-thirds of the alcohol-related deaths for both men and women. Approximately 18 per cent of alcohol-related deaths were assigned to fibrosis and cirrhosis of the liver for both men and women.

The numbers of alcohol-related deaths by NS-SEC classification and by region are presented in Tables 4 and 5 , along with the numbers and percentages of deaths classified to occupied NS-SEC classes. 


\section{Table $3 \quad$ Number of deaths attributed to specific alcohol-related causes, men aged 25-64 and women aged 25-59, England and Wales, 2001-03}

\begin{tabular}{|c|c|c|c|c|c|}
\hline $\begin{array}{l}\text { ICD } 10 \\
\text { CODE }\end{array}$ & Description & $\begin{array}{c}\text { Number of } \\
\text { deaths (Men) }\end{array}$ & $\begin{array}{l}\text { Percent of alcohol-related } \\
\text { deaths attributed to specific } \\
\text { alcohol-related causes } \\
\text { (Men) }\end{array}$ & $\begin{array}{l}\text { Number of } \\
\text { deaths } \\
\text { (Women) }\end{array}$ & $\begin{array}{l}\text { Percent of alcohol-related } \\
\text { deaths attributed to specific } \\
\text { alcohol-related causes } \\
\text { (Women) }\end{array}$ \\
\hline K70 & Alcoholic liver disease & 5,939 & 66.4 & 2,472 & 68.4 \\
\hline K74 & $\begin{array}{l}\text { Fibrosis and cirrhosis of } \\
\text { liver (Excluding K74.3- } \\
\text { K74.5: } \\
\text { Biliary cirrhosis) }\end{array}$ & 1,582 & 17.7 & 658 & 18.2 \\
\hline F10 & $\begin{array}{l}\text { Mental and behavioural } \\
\text { disorders due to use of } \\
\text { alcohol }\end{array}$ & 841 & 9.4 & 295 & 8.2 \\
\hline $\begin{array}{l}\mathrm{X} 45, \mathrm{X} 65 \\
\text { and } \mathrm{Y} 15\end{array}$ & $\begin{array}{l}\text { Accidental poisoning and } \\
\text { exposure to alcohol, } \\
\text { Intentional self-poisoning } \\
\text { by and exposure to } \\
\text { alcohol and Poisoning by } \\
\text { and exposure to alcohol, } \\
\text { intent undetermined }\end{array}$ & 265 & 3 & 96 & 2.7 \\
\hline 142.6 & $\begin{array}{l}\text { Alcoholic } \\
\text { cardiomyopathy }\end{array}$ & 221 & 2.5 & 37 & 1 \\
\hline K86.0 & $\begin{array}{l}\text { Alcoholic induced } \\
\text { chronic pancreatitis }\end{array}$ & 60 & 0.7 & 23 & 0.6 \\
\hline G31.2 & $\begin{array}{l}\text { Degeneration of nervous } \\
\text { system due to alcohol }\end{array}$ & 15 & 0.2 & 3 & 0.1 \\
\hline K73 & $\begin{array}{l}\text { Chronic hepatitis, not } \\
\text { elsewhere classified }\end{array}$ & 12 & 0.1 & 25 & 0.7 \\
\hline K29.2 & Alcoholic gastritis & 3 & 0.03 & 4 & 0.1 \\
\hline \multirow[t]{2}{*}{ G62.1 } & Alcoholic polyneuropathy & 0 & 0 & 0 & 0 \\
\hline & Total & 8,938 & 100 & 3,613 & 100 \\
\hline
\end{tabular}



classification and by region, men aged 25-64, 2001-03

\begin{tabular}{|c|c|c|c|c|c|c|c|c|c|c|c|}
\hline NS-SEC analytic classes & \multirow{2}{*}{$\begin{array}{r}\begin{array}{r}\text { England } \\
\text { and } \\
\text { Wales }^{1}\end{array} \\
603\end{array}$} & \multirow{2}{*}{$\begin{array}{r}\text { North } \\
\text { East } \\
22\end{array}$} & \multirow{2}{*}{$\begin{array}{r}\text { North } \\
\text { West } \\
96\end{array}$} & \multirow{2}{*}{$\begin{array}{r}\begin{array}{r}\text { Yorkshire } \\
\text { and The } \\
\text { Humber }\end{array} \\
42\end{array}$} & \multirow{2}{*}{$\begin{array}{r}\text { East } \\
\text { Midlands }\end{array}$} & \multirow{2}{*}{$\begin{array}{r}\begin{array}{r}\text { West } \\
\text { Midlands }\end{array} \\
88\end{array}$} & \multirow{2}{*}{$\begin{array}{r}\begin{array}{r}\text { East of } \\
\text { England }\end{array} \\
56\end{array}$} & \multirow{2}{*}{$\begin{array}{r}\text { London } \\
85\end{array}$} & \multirow{2}{*}{$\begin{array}{r}\begin{array}{r}\text { South } \\
\text { East }\end{array} \\
90\end{array}$} & \multirow{2}{*}{$\begin{array}{r}\begin{array}{r}\text { South } \\
\text { West }\end{array} \\
49\end{array}$} & \multirow{2}{*}{$\begin{array}{r}\text { Wales } \\
23\end{array}$} \\
\hline 1 Higher managerial and professional & & & & & & & & & & & \\
\hline 1.1 Large employers and higher managerial & 207 & 8 & 34 & 18 & 16 & 36 & 23 & 20 & 25 & 18 & 5 \\
\hline 1.2 Higher professional & 396 & 14 & 62 & 24 & 27 & 52 & 33 & 65 & 65 & 31 & 18 \\
\hline 2 Lower managerial and professional & 1,282 & 73 & 189 & 102 & 90 & 143 & 101 & 177 & 198 & 135 & 71 \\
\hline 3 Intermediate & 347 & 25 & 60 & 21 & 22 & 29 & 28 & 59 & 55 & 24 & 24 \\
\hline 4 Small employers and own account workers & 1,122 & 36 & 168 & 80 & 73 & 122 & 93 & 192 & 182 & 109 & 55 \\
\hline 5 Lower supervisory and technical & 1,115 & 84 & 209 & 110 & 79 & 142 & 75 & 114 & 142 & 83 & 71 \\
\hline 6 Semi-routine & 1,250 & 60 & 255 & 116 & 90 & 169 & 88 & 165 & 145 & 88 & 69 \\
\hline 7 Routine & 1,875 & 128 & 369 & 220 & 124 & 277 & 108 & 182 & 205 & 126 & 133 \\
\hline Non-occupied ${ }^{2}$ & 1,344 & 71 & 234 & 97 & 85 & 126 & 75 & 323 & 163 & 81 & 82 \\
\hline Total & 8,938 & 499 & 1,580 & 788 & 606 & 1,096 & 624 & 1,297 & 1,180 & 695 & 528 \\
\hline Number of deaths classified to occupied NS-SEC classes & 7,594 & 428 & 1,346 & 691 & 521 & 970 & 549 & 974 & 1,017 & 614 & 446 \\
\hline Percent of deaths classified to occupied NS-SEC classes & 85 & 86 & 85 & 88 & 86 & 89 & 88 & 75 & 86 & 88 & 84 \\
\hline
\end{tabular}

1 Figures for England \& Wales as a whole but not Wales and regions of England include deaths of non-residents 2 The 'Non-occupied' category includes 'Never worked, long-term unemployed, full-time students, inadequately described and unclassified for other reasons'

Source: ONS

\section{Table $5 \quad$ Adjusted number of alcohol-related deaths by NS-SEC and by region, women aged 25-59, 2001-03}

\begin{tabular}{|c|c|c|c|c|c|c|c|c|c|c|c|}
\hline NS-SEC analytic classes & \multirow{2}{*}{$\begin{array}{r}\begin{array}{r}\text { England } \\
\text { and } \\
\text { Wales }^{1}\end{array} \\
239\end{array}$} & \multirow{2}{*}{$\begin{array}{r}\begin{array}{r}\text { North } \\
\text { East }\end{array} \\
15\end{array}$} & \multirow{2}{*}{$\begin{array}{r}\begin{array}{r}\text { North } \\
\text { West }\end{array} \\
41\end{array}$} & \multirow{2}{*}{$\begin{array}{r}\begin{array}{r}\text { Yorkshire } \\
\text { and The } \\
\text { Humber }\end{array} \\
15\end{array}$} & \multirow{2}{*}{$\begin{array}{r}\begin{array}{r}\text { East } \\
\text { Midlands }\end{array} \\
18\end{array}$} & \multirow{2}{*}{$\begin{array}{r}\begin{array}{r}\text { West } \\
\text { Midlands }\end{array} \\
20\end{array}$} & \multirow{2}{*}{$\begin{array}{r}\begin{array}{r}\text { East of } \\
\text { England }\end{array} \\
31\end{array}$} & \multirow{2}{*}{$\begin{array}{r}\text { London } \\
24\end{array}$} & \multirow{2}{*}{$\begin{array}{r}\begin{array}{r}\text { South } \\
\text { East }\end{array} \\
36\end{array}$} & \multirow{2}{*}{$\begin{array}{r}\begin{array}{r}\text { South } \\
\text { West }\end{array} \\
23\end{array}$} & \multirow{2}{*}{$\begin{array}{r}\text { Wales } \\
13\end{array}$} \\
\hline 1 Higher managerial and professional & & & & & & & & & & & \\
\hline 1.1 Large employers and higher managerial & 124 & 8 & 20 & 7 & 11 & 16 & 13 & 12 & 15 & 13 & 7 \\
\hline 1.2 Higher professional & 116 & 6 & 21 & 7 & 6 & 5 & 17 & 12 & 21 & 11 & 6 \\
\hline 2 Lower managerial and professional & 709 & 54 & 120 & 65 & 50 & 65 & 52 & 83 & 117 & 61 & 40 \\
\hline 3 Intermediate & 427 & 37 & 75 & 40 & 30 & 46 & 36 & 57 & 56 & 22 & 27 \\
\hline 4 Small employers and own account workers & 289 & 15 & 59 & 18 & 21 & 34 & 19 & 35 & 37 & 24 & 23 \\
\hline 5 Lower supervisory and technical & 376 & 31 & 74 & 37 & 31 & 48 & 19 & 33 & 44 & 39 & 19 \\
\hline 6 Semi-routine & 668 & 38 & 149 & 59 & 44 & 83 & 40 & 78 & 72 & 57 & 44 \\
\hline 7 Routine & 578 & 37 & 134 & 55 & 44 & 76 & 38 & 56 & 51 & 39 & 44 \\
\hline Non-occupied ${ }^{2}$ & 327 & 26 & 65 & 25 & 25 & 33 & 21 & 51 & 37 & 23 & 20 \\
\hline Total & 3613 & 253 & 717 & 314 & 263 & 406 & 256 & 416 & 450 & 288 & 230 \\
\hline Number of deaths classified to occupied NS-SEC classes & 3,286 & 227 & 652 & 289 & 238 & 373 & 235 & 365 & 413 & 265 & 210 \\
\hline Percent of deaths classified to occupied NS-SEC classes & 91 & 90 & 91 & 92 & 91 & 92 & 92 & 88 & 92 & 92 & 91 \\
\hline
\end{tabular}

1 Figures for England \& Wales as a whole but not Wales and regions of England include deaths of non-residents 2 The 'Non-occupied' category includes 'Never worked, long-term unemployed, full-time students, inadequately described and unclassified for other reasons'

Source: ONS 
England and Wales: Age-specific and age-standardised mortality rates by NS-SEC

Age-specific mortality rates by NS-SEC

The age-specific mortality rates per 100,000 by five-year age group and by NS-SEC are presented in Table 6 (men) and Table 7 (women), and illustrated in Figure 1 (men) and on Figure 2 (women).

Figure $1 \quad$ Age-specific mortality rates from alcohol-related causes of death by five-year age group and NS-SEC, men aged 25-64, England and Wales, 2001-03

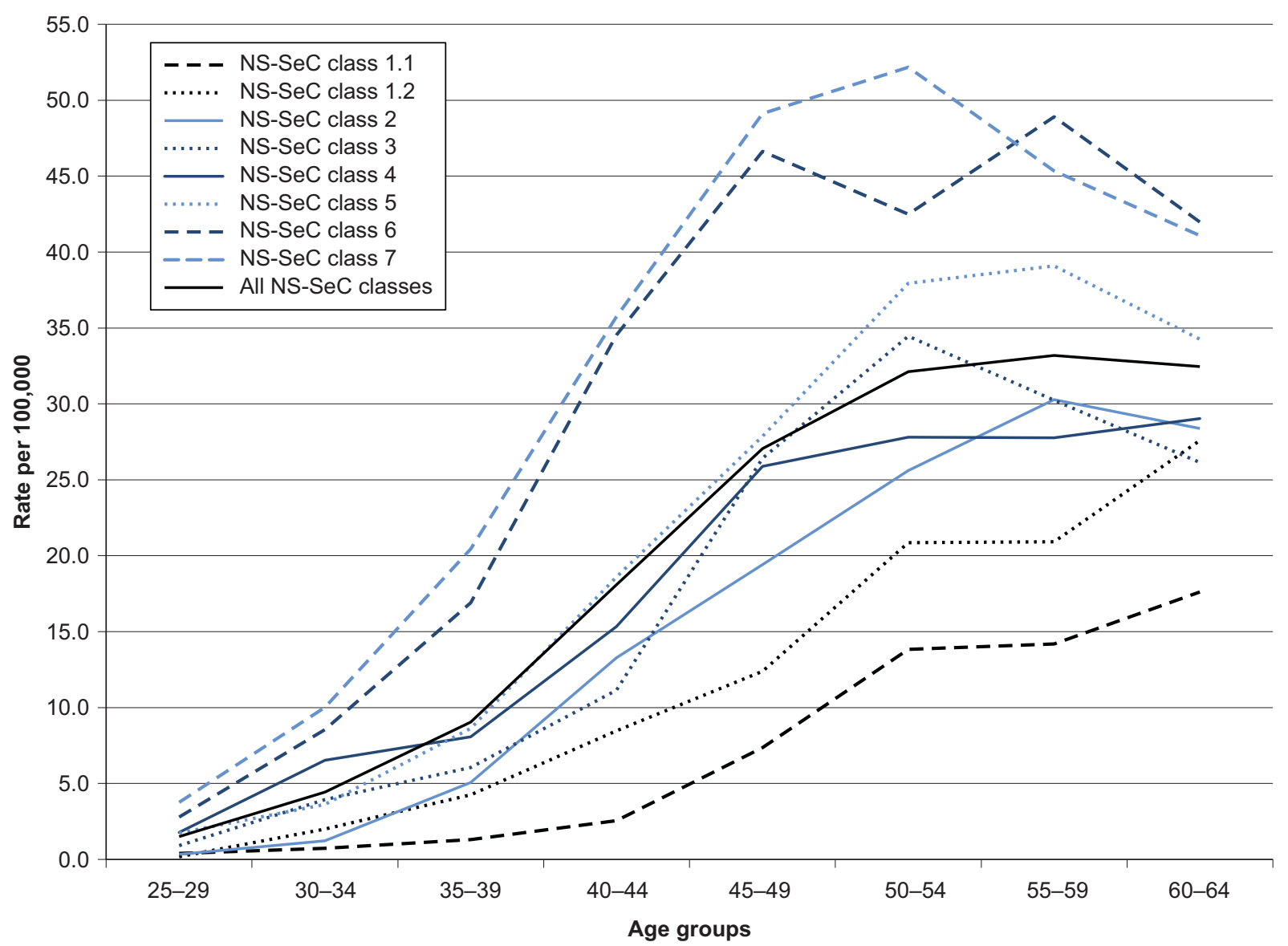

Source: ONS 


\section{Table 6 Age-specific mortality rates ${ }^{1,2,3}$ by five year age group and NS-SEC classification, men aged 25-64, England and Wales, 2001-03}

Rate per 100,000

\begin{tabular}{|c|c|c|c|c|c|c|c|c|c|c|}
\hline \multirow{2}{*}{\multicolumn{2}{|c|}{ NS-SEC analytic classes }} & \multicolumn{9}{|c|}{ Age (years) } \\
\hline & & $25-29$ & $30-34$ & $35-39$ & $40-44$ & $45-49$ & $50-54$ & $55-59$ & $60-64$ & 25-64 \\
\hline 1 & Higher managerial and professional & $(:)$ & $\begin{array}{r}1.5 \\
(0.9,2.5)\end{array}$ & $\begin{array}{r}2.8 \\
(2.0,4.0)\end{array}$ & $\begin{array}{r}5.5 \\
(4.2,7.1)\end{array}$ & $\begin{array}{r}9.8 \\
(8.0,12.1)\end{array}$ & $\begin{array}{r}17.4 \\
(14.9,20.4)\end{array}$ & $\begin{array}{r}17.7 \\
(15.0,20.9)\end{array}$ & $\begin{array}{r}23.5 \\
(19.6,28.1)\end{array}$ & $\begin{array}{r}8.5 \\
(7.9,9.2)\end{array}$ \\
\hline 1.1 & Large employers and higher managerial & $(:)$ & $\begin{array}{r}0.7 \\
(0.2,2.3)\end{array}$ & $\begin{array}{r}1.3 \\
(0.6,2.7)\end{array}$ & $\begin{array}{r}2.6 \\
(1.5,4.4)\end{array}$ & $\begin{array}{r}7.4 \\
(5.3,10.3)\end{array}$ & $\begin{array}{r}13.8 \\
(10.7,17.9)\end{array}$ & $\begin{array}{r}14.2 \\
(10.8,18.6)\end{array}$ & $\begin{array}{r}17.6 \\
(12.8,24.3)\end{array}$ & $\begin{array}{r}6.5 \\
(5.7,7.5)\end{array}$ \\
\hline 1.2 & Higher professional & $(:)$ & $\begin{array}{r}2.0 \\
(1.2,3.4)\end{array}$ & $\begin{array}{r}4.3 \\
(2.9,6.3)\end{array}$ & $\begin{array}{r}8.5 \\
(6.3,11.5)\end{array}$ & $\begin{array}{r}12.4 \\
(9.5,16.1)\end{array}$ & $\begin{array}{r}20.9 \\
(17.0,25.6)\end{array}$ & $\begin{array}{r}20.9 \\
(16.9,25.9)\end{array}$ & $\begin{array}{r}27.6 \\
(22.2,34.3)\end{array}$ & $\begin{array}{r}10.2 \\
(9.2,11.2)\end{array}$ \\
\hline 2 & Lower managerial \& professional & $\begin{array}{r}0.3 \\
(0.1,0.9)\end{array}$ & $\begin{array}{r}1.2 \\
(0.8,2.0)\end{array}$ & $\begin{array}{r}5.1 \\
(4.0,6.4)\end{array}$ & $\begin{array}{r}13.3 \\
(11.4,15.4)\end{array}$ & $\begin{array}{r}19.4 \\
(17.0,22.2)\end{array}$ & $\begin{array}{r}25.6 \\
(22.9,28.7)\end{array}$ & $\begin{array}{r}30.3 \\
(27.1,33.9)\end{array}$ & $\begin{array}{r}28.4 \\
(24.7,32.5)\end{array}$ & $\begin{array}{r}13.9 \\
(13.2,14.7)\end{array}$ \\
\hline 3 & Intermediate & $\begin{array}{r}0.9 \\
(0.3,2.4)\end{array}$ & $\begin{array}{r}3.9 \\
(2.5,6.3)\end{array}$ & $\begin{array}{r}6.1 \\
(4.0,9.3)\end{array}$ & $\begin{array}{r}11.1 \\
(7.9,15.7)\end{array}$ & $\begin{array}{r}26.4 \\
(20.8,33.6)\end{array}$ & $\begin{array}{r}34.4 \\
(28.0,42.4)\end{array}$ & $\begin{array}{r}30.2 \\
(23.9,38.3)\end{array}$ & $\begin{array}{r}26.1 \\
(19.6,34.9)\end{array}$ & $\begin{array}{r}14.2 \\
(12.8,15.8)\end{array}$ \\
\hline 4 & Small employers \& own account workers & $\begin{array}{r}1.8 \\
(0.8,4.0)\end{array}$ & $\begin{array}{r}6.5 \\
(4.7,9.0)\end{array}$ & $\begin{array}{r}8.1 \\
(6.4,10.3)\end{array}$ & $\begin{array}{r}15.3 \\
(12.9,18.2)\end{array}$ & $\begin{array}{r}25.9 \\
(22.6,29.7)\end{array}$ & $\begin{array}{r}27.8 \\
(24.5,31.5)\end{array}$ & $\begin{array}{r}27.8 \\
(24.5,31.5)\end{array}$ & $\begin{array}{r}29.0 \\
(25.3,33.4)\end{array}$ & $\begin{array}{r}19.4 \\
(18.3,20.6)\end{array}$ \\
\hline 5 & Lower supervisory and technical & $\begin{array}{r}1.7 \\
(1.0,3.1)\end{array}$ & $\begin{array}{r}3.6 \\
(2.6,5.1)\end{array}$ & $\begin{array}{r}8.6 \\
(6.9,10.8)\end{array}$ & $\begin{array}{r}18.6 \\
(15.8,21.9)\end{array}$ & $\begin{array}{r}27.9 \\
(24.1,32.2)\end{array}$ & $\begin{array}{r}37.9 \\
(33.5,43.0)\end{array}$ & $\begin{array}{r}39.1 \\
(34.4,44.4)\end{array}$ & $\begin{array}{r}34.3 \\
(29.6,39.6)\end{array}$ & $\begin{array}{r}19.7 \\
(18.6,20.9)\end{array}$ \\
\hline 6 & Semi-routine & $\begin{array}{r}2.8 \\
(1.8,4.4)\end{array}$ & $\begin{array}{r}8.6 \\
(6.6,11.1)\end{array}$ & $\begin{array}{r}16.9 \\
(14.0,20.4)\end{array}$ & $\begin{array}{r}34.5 \\
(30.1,39.7)\end{array}$ & $\begin{array}{r}46.6 \\
(41.0,53.0)\end{array}$ & $\begin{array}{r}42.5 \\
(37.2,48.6)\end{array}$ & $\begin{array}{r}48.9 \\
(43.1,55.5)\end{array}$ & $\begin{array}{r}42.0 \\
(36.3,48.6)\end{array}$ & $\begin{array}{r}28.1 \\
(26.6,29.7)\end{array}$ \\
\hline 7 & Routine & $\begin{array}{r}3.8 \\
(2.6,5.5)\end{array}$ & $\begin{array}{r}10.0 \\
(8.1,12.4)\end{array}$ & $\begin{array}{r}20.4 \\
(17.6,23.7)\end{array}$ & $\begin{array}{r}35.7 \\
(31.7,40.3)\end{array}$ & $\begin{array}{r}49.1 \\
(44.1,54.7)\end{array}$ & $\begin{array}{r}52.2 \\
(47.2,57.7)\end{array}$ & $\begin{array}{r}45.3 \\
(40.8,50.5)\end{array}$ & $\begin{array}{r}41.1 \\
(36.5,46.2)\end{array}$ & $\begin{array}{r}31.4 \\
(30.0,32.9)\end{array}$ \\
\hline & All classes & $\begin{array}{r}1.5 \\
(1.2,1.9)\end{array}$ & $\begin{array}{r}4.4 \\
(3.9,5.0)\end{array}$ & $\begin{array}{r}9.0 \\
(8.3,9.8)\end{array}$ & $\begin{array}{r}18.1 \\
(17.0,19.3)\end{array}$ & $\begin{array}{r}27.1 \\
(25.6,28.6)\end{array}$ & $\begin{array}{r}32.1 \\
(30.6,33.7)\end{array}$ & $\begin{array}{r}33.2 \\
(31.6,34.9)\end{array}$ & $\begin{array}{r}32.5 \\
(30.7,34.3)\end{array}$ & $\begin{array}{r}18.7 \\
(18.3,19.1)\end{array}$ \\
\hline & Ratio 7:1 & $\begin{array}{r}15.5 \\
(3.7,65.0)\end{array}$ & $\begin{array}{r}6.6 \\
(3.9,11.3)\end{array}$ & $\begin{array}{r}7.2 \\
(4.9,10.5)\end{array}$ & $\begin{array}{r}6.5 \\
(4.9,8.7)\end{array}$ & $\begin{array}{r}5.0 \\
(4.0,6.3)\end{array}$ & $\begin{array}{r}3.0 \\
(2.5,3.6)\end{array}$ & $\begin{array}{r}2.6 \\
(2.1,3.1)\end{array}$ & $\begin{array}{r}1.8 \\
(1.4,2.2)\end{array}$ & $\begin{array}{r}3.7 \\
(3.4,4.0)\end{array}$ \\
\hline
\end{tabular}

195 per cent confidence intervals shown in parenthesis

2 Rates were not calculated where there were fewer than 3 deaths in a cell, denoted by $(:)$

3. Rates calculated from fewer than 20 deaths are shown in italics

Source: ONS 
Figure 2 Age-specific mortality rates from alcohol-related causes of death by five-year age group and NS-SEC, women aged 2559, England and Wales, 2001-03

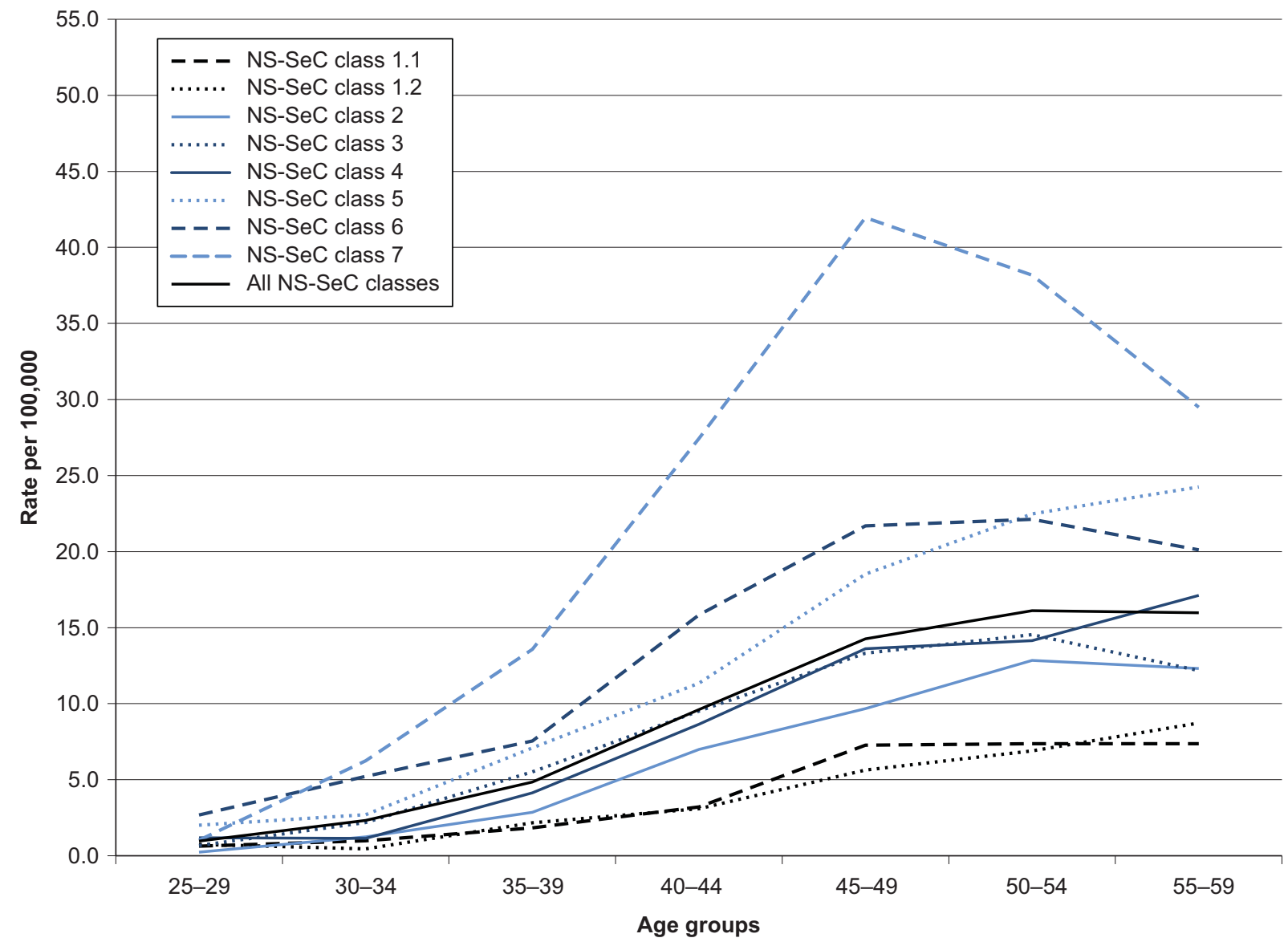

Source: ONS 


\section{Table $7 \quad$ Age-specific mortality rates ${ }^{1,2,3}$ by five year age group and NS-SEC classification, women aged 25-59, England and Wales, 2001-03}

Rate per 100,000

\begin{tabular}{|c|c|c|c|c|c|c|c|c|c|}
\hline \multirow{2}{*}{\multicolumn{2}{|c|}{ NS-SEC analytic classes }} & \multicolumn{8}{|c|}{ Age (years) } \\
\hline & & $25-29$ & $30-34$ & $35-39$ & $40-44$ & $45-49$ & $50-54$ & $55-59$ & $25-59$ \\
\hline 1 & Higher managerial and professional & $\begin{array}{r}0.7 \\
(0.3,1.7)\end{array}$ & $\begin{array}{r}0.7 \\
(0.4,1.5)\end{array}$ & $\begin{array}{r}2.0 \\
(1.3,3.0)\end{array}$ & $\begin{array}{r}3.2 \\
(2.2,4.4)\end{array}$ & $\begin{array}{r}6.5 \\
(5.0,8.4)\end{array}$ & $\begin{array}{r}7.1 \\
(5.5,9.2)\end{array}$ & $\begin{array}{r}8.1 \\
(6.2,10.6)\end{array}$ & $\begin{array}{r}3.7 \\
(3.3,4.3)\end{array}$ \\
\hline 1.1 & Large employers and higher managerial & $(:)$ & $\begin{array}{r}1.0 \\
(0.4,2.3)\end{array}$ & $\begin{array}{r}1.8 \\
(1.0,3.3)\end{array}$ & $\begin{array}{r}3.2 \\
(2.0,5.1)\end{array}$ & $\begin{array}{r}7.3 \\
(5.2,10.2)\end{array}$ & $\begin{array}{r}7.4 \\
(5.2,10.5)\end{array}$ & $\begin{array}{r}7.4 \\
(4.9,11.1)\end{array}$ & $\begin{array}{r}3.9 \\
(3.2,4.6)\end{array}$ \\
\hline 1.2 & Higher professional & $\begin{array}{r}0.7 \\
(0.2,2.2)\end{array}$ & $(:)$ & $\begin{array}{r}2.2 \\
(1.2,3.8)\end{array}$ & $\begin{array}{r}3.1 \\
(1.9,5.1)\end{array}$ & $\begin{array}{r}5.6 \\
(3.8,8.4)\end{array}$ & $\begin{array}{r}6.9 \\
(4.8,10.0)\end{array}$ & $\begin{array}{r}8.7 \\
(6.1,12.5)\end{array}$ & $\begin{array}{r}3.6 \\
(3.0,4.3)\end{array}$ \\
\hline 2 & Lower managerial \& professional & $\begin{array}{r}0.2 \\
(0.1,0.7)\end{array}$ & $\begin{array}{r}1.2 \\
(0.8,1.9)\end{array}$ & $\begin{array}{r}2.8 \\
(2.2,3.7)\end{array}$ & $\begin{array}{r}7.0 \\
(5.8,8.4)\end{array}$ & $\begin{array}{r}9.7 \\
(8.2,11.3)\end{array}$ & $\begin{array}{r}12.8 \\
(11.2,14.8)\end{array}$ & $\begin{array}{r}12.3 \\
(10.6,14.4)\end{array}$ & $\begin{array}{r}6.3 \\
(5.8,6.8)\end{array}$ \\
\hline 3 & Intermediate & $\begin{array}{r}0.7 \\
(0.3,1.5)\end{array}$ & $\begin{array}{r}2.2 \\
(1.4,3.4)\end{array}$ & $\begin{array}{r}5.5 \\
(4.2,7.3)\end{array}$ & $\begin{array}{r}9.5 \\
(7.6,12.0)\end{array}$ & $\begin{array}{r}13.3 \\
(10.8,16.4)\end{array}$ & $\begin{array}{r}14.5 \\
(12.0,17.6)\end{array}$ & $\begin{array}{r}12.2 \\
(9.9,15.1)\end{array}$ & $\begin{array}{r}7.8 \\
(7.1,8.6)\end{array}$ \\
\hline 4 & Small employers \& own account workers & $(:)$ & $\begin{array}{r}1.1 \\
(0.4,3.0)\end{array}$ & $\begin{array}{r}4.1 \\
(2.6,6.5)\end{array}$ & $\begin{array}{r}8.6 \\
(6.4,11.8)\end{array}$ & $\begin{array}{r}13.6 \\
(10.6,17.5)\end{array}$ & $\begin{array}{r}14.2 \\
(11.3,17.8)\end{array}$ & $\begin{array}{r}17.1 \\
(13.9,21.1)\end{array}$ & $\begin{array}{r}9.8 \\
(8.7,11.0)\end{array}$ \\
\hline 5 & Lower supervisory and technical & $\begin{array}{r}2.0 \\
(0.9,4.4)\end{array}$ & $\begin{array}{r}2.7 \\
(1.5,4.9)\end{array}$ & $\begin{array}{r}7.1 \\
(5.0,10.0)\end{array}$ & $\begin{array}{r}11.3 \\
(8.6,15.0)\end{array}$ & $\begin{array}{r}18.5 \\
(14.7,23.3)\end{array}$ & $\begin{array}{r}22.5 \\
(18.4,27.4)\end{array}$ & $\begin{array}{r}24.2 \\
(20.0,29.3)\end{array}$ & $\begin{array}{r}13.0 \\
(11.8,14.4)\end{array}$ \\
\hline 6 & Semi-routine & $\begin{array}{r}2.7 \\
(1.7,4.2)\end{array}$ & $\begin{array}{r}5.2 \\
(3.9,7.1)\end{array}$ & $\begin{array}{r}7.5 \\
(5.9,9.7)\end{array}$ & $\begin{array}{r}15.8 \\
(13.2,19.0)\end{array}$ & $\begin{array}{r}21.7 \\
(18.3,25.7)\end{array}$ & $\begin{array}{r}22.1 \\
(18.9,26.0)\end{array}$ & $\begin{array}{r}20.1 \\
(17.1,23.6)\end{array}$ & $\begin{array}{r}13.2 \\
(12.2,14.3)\end{array}$ \\
\hline 7 & Routine & $\begin{array}{r}1.0 \\
(0.4,2.8)\end{array}$ & $\begin{array}{r}6.2 \\
(4.3,9.1)\end{array}$ & $\begin{array}{r}13.6 \\
(10.5,17.6)\end{array}$ & $\begin{array}{r}27.4 \\
(22.6,33.4)\end{array}$ & $\begin{array}{r}42.0 \\
(35.4,49.7)\end{array}$ & $\begin{array}{r}38.2 \\
(32.3,45.2)\end{array}$ & $\begin{array}{r}29.5 \\
(24.6,35.3)\end{array}$ & $\begin{array}{r}21.7 \\
(20.0,23.6)\end{array}$ \\
\hline & All classes & $\begin{array}{r}1.0 \\
(0.7,1.3)\end{array}$ & $\begin{array}{r}2.3 \\
(2.0,2.8)\end{array}$ & $\begin{array}{r}4.9 \\
(4.3,5.4)\end{array}$ & $\begin{array}{r}9.6 \\
(8.8,10.5)\end{array}$ & $\begin{array}{r}14.3 \\
(13.2,15.4)\end{array}$ & $\begin{array}{r}16.1 \\
(15.1,17.3)\end{array}$ & $\begin{array}{r}16.0 \\
(14.9,17.2)\end{array}$ & $\begin{array}{r}8.9 \\
(8.6,9.3)\end{array}$ \\
\hline & Ratio $7: 1$ & $\begin{array}{r}1.5 \\
(0.4,5.7)\end{array}$ & $\begin{array}{r}8.7 \\
(3.9,19.4)\end{array}$ & $\begin{array}{r}6.8 \\
(4.2,11.1)\end{array}$ & $\begin{array}{r}8.7 \\
(5.9,12.9)\end{array}$ & $\begin{array}{r}6.5 \\
(4.8,8.8)\end{array}$ & $\begin{array}{r}5.4 \\
(3.9,7.3)\end{array}$ & $\begin{array}{r}3.6 \\
(2.6,5.0)\end{array}$ & $\begin{array}{r}5.8 \\
(5.0,6.7)\end{array}$ \\
\hline
\end{tabular}

195 per cent confidence intervals shown in parenthesis

2 Rates were not calculated where there were fewer than 3 deaths in a cell, denoted by $(:)$

3 Rates calculated from fewer than 20 deaths are shown in italics

Source: ONS

\section{Age-specific mortality rates by NS-SEC - Men}

Men in Semi-routine (Class 6) and Routine (Class 7) occupations had markedly higher death rates from alcohol than men in other classes in each of the age groups studied, especially at younger ages. Routine workers aged 30-44 were about seven times more likely to die from alcohol-related causes than men of the same age in Higher managerial and professional occupations (Class 1). A higher mortality rate for Higher managers and professionals (Class 1) combined with a lower one for Routine occupations (Class 7) at ages 60-64 resulted in reduced inequalities in that age group compared to younger ages (Figure 1). The highest age-specific alcohol-related mortality rate in this study $(52.2$ per 100,000) was observed for men in Routine occupations (Class 7 ) aged 50-54.

For men in Intermediate occupations (Class 3 ) and Routine workers (Class 7), the alcohol-related mortality rates peaked at ages 50-54. For Lower managers and professionals (Class 2), Lower supervisory, technical workers (Class 5 ) and men in Semi-routine occupations (Class 6 ), the highest mortality rate from alcohol occurred at age 55-59.

Alcohol-related mortality rates for Small employers and own account workers (Class 4) also increased until ages $45-49$, but levelled out at older ages. 
The age-specific mortality rates of the two sub-groups of the Higher managerial and professional occupations (Class 1) differed, with Large employers and higher managers (Class 1.1) having a lower mortality rate from alcohol than Higher professionals (Class 1.2) at most ages. For both subgroups, the mortality rate from alcohol-related causes increased continuously between ages 25 and 64 , with a plateau in mortality between the ages of 50 and 59, but an increase at ages 60-64. However, Large employers and higher managers (Class 1.1) aged 55-64 had a lower alcoholrelated mortality than Routine class workers (Class 7 ) who were 20 years their junior.

\section{Age-specific mortality rates by NS-SEC - Women}

At ages 35-59, the mortality rates of women in Routine occupations (Class 7 ) were much higher than in any other classes. The mortality rates for women in Routine occupations (Class 7) were lower than for women in Semi-routine occupations (Class 6) at ages 25-29 and similar at ages 3034. Women in Routine occupations aged 30-44 were between 7 and 9 times more likely to die from alcohol-related causes than women in Higher managerial and professional occupations. (Class 1). The difference reduced to around four times at ages 55-59.

The highest mortality rate of all classes and age groups for women $(42.0$ per 100,000) occurred for Routine workers (Class 7 ) aged 45-49, (Figure 2). This compares with the highest age-specific rate for men, which was observed at age 50-54, as reported above.

The gap in mortality between Lower supervisory and technical workers (Class 5) and Semi-routine workers (Class 6) was narrower for women than for men. At ages 35-39, 50-54, and 55-59 women in these classes experienced similar mortality rates.

\section{Age-standardised mortality rates by NS-SEC}

The age-standardised mortality rates per 100,000 men aged 25-64, by NS-SEC class are presented in Table 8 and Figure 3. The corresponding rates for women aged 25-59 are found in Table 9 and Figure 3. The socio-economic gradients or ratios of mortality rates of men (or women) in Routine occupations (Class 7) to that of men (or women) in Higher managerial and professional occupations (Class 1) are also presented in Tables 8 and 9. 
Figure 3 Age-standardised mortality rates ${ }^{1}$ from alcohol-related causes of death by NS-SEC, men aged 25-64 and women aged 25-59, England and Wales, 2001-03

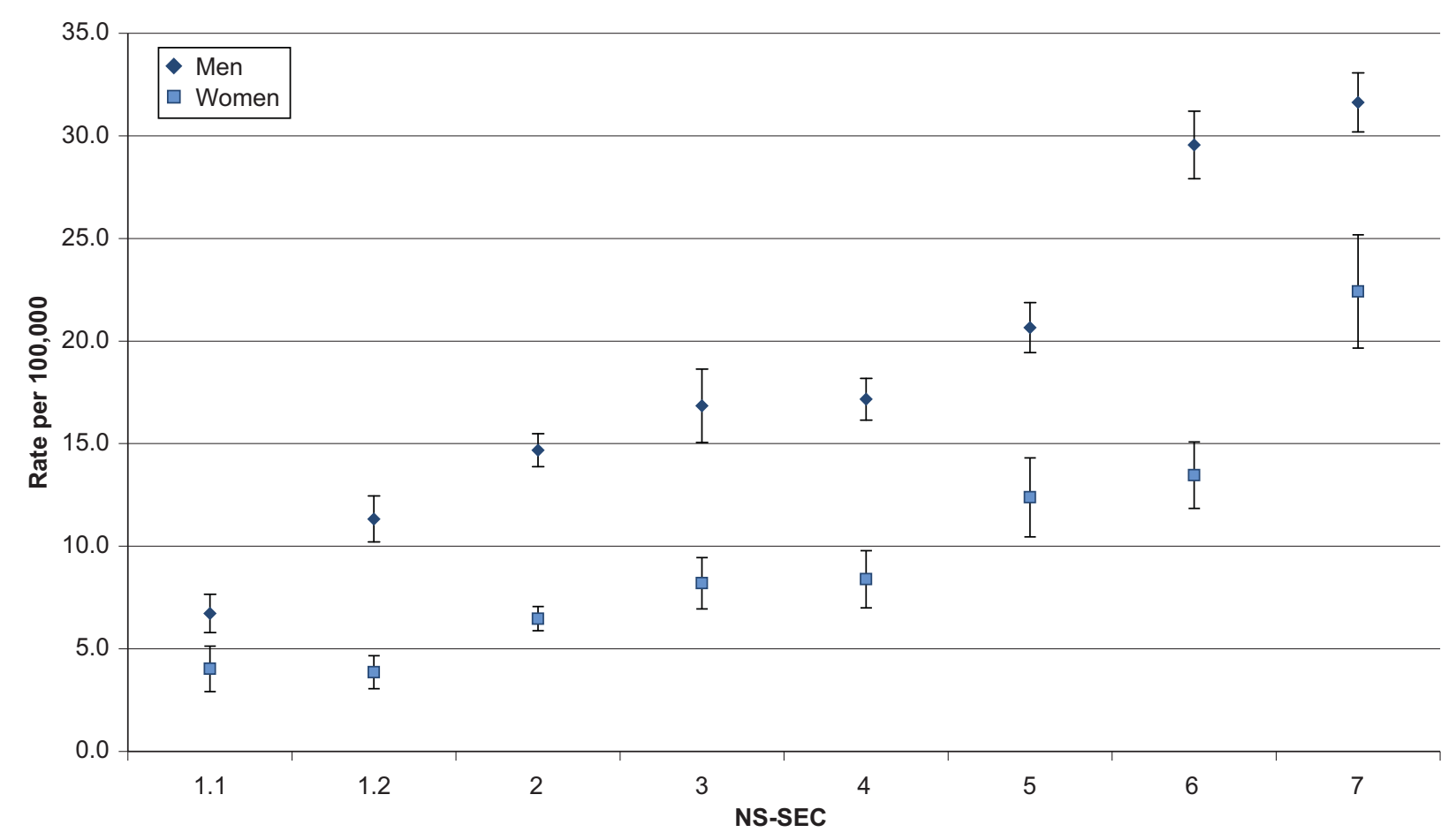

1 Directly age-standardised rates using the European standard population

Source: ONS 


\section{Table 8 Age-standardised mortality rates ${ }^{1,2,3,4}$ from alcohol-related causes of death, by NS-SEC classification and region, men aged 25-64, England and Wales, 2001-03}

Rate per 100,000

\begin{tabular}{|c|c|c|c|c|c|c|c|c|c|c|c|c|}
\hline \multirow{2}{*}{\multicolumn{2}{|c|}{ NS-SEC analytic classes }} & \multicolumn{11}{|c|}{ Regions of England and Wales } \\
\hline & & $\begin{array}{r}\text { England } \\
\text { and } \\
\text { Wales }\end{array}$ & $\begin{array}{r}\text { North } \\
\text { East }\end{array}$ & $\begin{array}{l}\text { North } \\
\text { West }\end{array}$ & $\begin{array}{l}\text { Yorshire } \\
\text { and The } \\
\text { Humber }\end{array}$ & $\begin{array}{r}\text { East } \\
\text { Midlands }\end{array}$ & $\begin{array}{r}\text { West } \\
\text { Midlands }\end{array}$ & $\begin{array}{r}\text { East of } \\
\text { England }\end{array}$ & London & $\begin{array}{r}\text { South } \\
\text { East }\end{array}$ & $\begin{array}{l}\text { South } \\
\text { West }\end{array}$ & Wales \\
\hline 1 & Higher managerial and professional & $\begin{array}{r}9.1 \\
(8.4,9.9)\end{array}$ & $\begin{array}{r}9.7 \\
(5.7,13.8)\end{array}$ & $\begin{array}{r}13.0 \\
(10.4,15.6)\end{array}$ & $\begin{array}{r}7.9 \\
(5.5,10.3)\end{array}$ & $\begin{array}{r}8.3 \\
(5.8,10.9)\end{array}$ & $\begin{array}{r}13.7 \\
(10.8,16.6)\end{array}$ & $\begin{array}{r}7.0 \\
(5.1,8.8)\end{array}$ & $\begin{array}{r}10.2 \\
(7.9,12.4)\end{array}$ & $\begin{array}{r}6.7 \\
(5.3,8.1)\end{array}$ & $\begin{array}{r}7.6 \\
(5.4,9.7)\end{array}$ & $\begin{array}{r}8.1 \\
(4.8,11.4)\end{array}$ \\
\hline 1.1 & Large employers and higher managerial & $\begin{array}{r}6.7 \\
(5.8,7.7)\end{array}$ & $(:)$ & $\begin{array}{r}9.7 \\
(6.4,13.0)\end{array}$ & $\begin{array}{r}6.8 \\
(3.6,10.0)\end{array}$ & $\begin{array}{r}6.0 \\
(3.0,8.9)\end{array}$ & $\begin{array}{r}10.8 \\
(7.2,14.3)\end{array}$ & $\begin{array}{r}5.9 \\
(3.4,8.3)\end{array}$ & $\begin{array}{r}6.4 \\
(3.5,9.3)\end{array}$ & $\begin{array}{r}4.0 \\
(2.4,5.6)\end{array}$ & $\begin{array}{r}6.3 \\
(3.4,9.3)\end{array}$ & $(:)$ \\
\hline 1.2 & Higher professional & $\begin{array}{r}11.3 \\
(10.2,12.5)\end{array}$ & $\begin{array}{r}11.5 \\
(5.5,17.6)\end{array}$ & $\begin{array}{r}15.9 \\
(12.0,19.9)\end{array}$ & $\begin{array}{r}8.9 \\
(5.3,12.5)\end{array}$ & $\begin{array}{r}10.6 \\
(6.6,14.6)\end{array}$ & $\begin{array}{r}17.0 \\
(12.3,21.6)\end{array}$ & $\begin{array}{r}8.1 \\
(5.3,10.9)\end{array}$ & $\begin{array}{r}12.7 \\
(9.5,15.9)\end{array}$ & $\begin{array}{r}9.1 \\
(6.9,11.4)\end{array}$ & $\begin{array}{r}8.7 \\
(5.6,11.7)\end{array}$ & $\begin{array}{r}11.1 \\
(6.0,16.3)\end{array}$ \\
\hline 2 & Lower managerial \& professional & $\begin{array}{r}14.7 \\
(13.9,15.5)\end{array}$ & $\begin{array}{r}19.9 \\
(15.3,24.5)\end{array}$ & $\begin{array}{r}18.6 \\
(15.9,21.2)\end{array}$ & $\begin{array}{r}13.5 \\
(10.9,16.2)\end{array}$ & $\begin{array}{r}13.2 \\
(10.5,15.9)\end{array}$ & $\begin{array}{r}17.9 \\
(14.9,20.8)\end{array}$ & $\begin{array}{r}9.8 \\
(7.9,11.7)\end{array}$ & $\begin{array}{r}16.0 \\
(13.6,18.4)\end{array}$ & $\begin{array}{r}12.3 \\
(10.6,14.0)\end{array}$ & $\begin{array}{r}15.0 \\
(12.5,17.5)\end{array}$ & $\begin{array}{r}16.1 \\
(12.3,19.8)\end{array}$ \\
\hline 3 & Intermediate & $\begin{array}{r}16.8 \\
(15.1,18.6)\end{array}$ & $\begin{array}{r}25.3 \\
(15.1,35.5)\end{array}$ & $\begin{array}{r}22.7 \\
(16.9,28.4)\end{array}$ & $\begin{array}{r}11.5 \\
(6.5,16.4)\end{array}$ & $\begin{array}{r}14.6 \\
(8.4,20.7)\end{array}$ & $\begin{array}{r}15.7 \\
(9.9,21.4)\end{array}$ & $\begin{array}{r}12.9 \\
(8.2,17.7)\end{array}$ & $\begin{array}{r}19.4 \\
(14.4,24.5)\end{array}$ & $\begin{array}{r}15.8 \\
(11.6,20.0)\end{array}$ & $\begin{array}{r}11.9 \\
(7.1,16.7)\end{array}$ & $\begin{array}{r}23.7 \\
(14.2,33.3)\end{array}$ \\
\hline 4 & Small employers \& own account workers & $\begin{array}{r}17.2 \\
(16.1,18.2)\end{array}$ & $\begin{array}{r}16.8 \\
(11.3,22.3)\end{array}$ & $\begin{array}{r}21.4 \\
(18.2,24.7)\end{array}$ & $\begin{array}{r}14.4 \\
(11.1,17.7)\end{array}$ & $\begin{array}{r}14.5 \\
(11.0,17.9)\end{array}$ & $\begin{array}{r}19.7 \\
(16.1,23.2)\end{array}$ & $\begin{array}{r}12.0 \\
(9.6,14.5)\end{array}$ & $\begin{array}{r}24.1 \\
(20.7,27.6)\end{array}$ & $\begin{array}{r}16.5 \\
(14.0,18.9)\end{array}$ & $\begin{array}{r}13.5 \\
(10.9,16.0)\end{array}$ & $\begin{array}{r}15.2 \\
(11.1,19.3)\end{array}$ \\
\hline 5 & Lower supervisory and technical & $\begin{array}{r}20.7 \\
(19.4,21.9)\end{array}$ & $\begin{array}{r}27.2 \\
(21.4,33.1)\end{array}$ & $\begin{array}{r}27.8 \\
(24.0,31.6)\end{array}$ & $\begin{array}{r}19.4 \\
(15.7,23.0)\end{array}$ & $\begin{array}{r}15.9 \\
(12.3,19.4)\end{array}$ & $\begin{array}{r}23.3 \\
(19.4,27.1)\end{array}$ & $\begin{array}{r}13.4 \\
(10.4,16.5)\end{array}$ & $\begin{array}{r}24.4 \\
(19.8,28.9)\end{array}$ & $\begin{array}{r}18.5 \\
(15.5,21.6)\end{array}$ & $\begin{array}{r}16.1 \\
(12.7,19.6)\end{array}$ & $\begin{array}{r}21.5 \\
(16.5,26.5)\end{array}$ \\
\hline 6 & Semi-routine & $\begin{array}{r}29.6 \\
(27.9,31.2)\end{array}$ & $\begin{array}{r}26.5 \\
(19.7,33.2)\end{array}$ & $\begin{array}{r}43.9 \\
(38.5,49.3)\end{array}$ & $\begin{array}{r}26.2 \\
(21.4,31.0)\end{array}$ & $\begin{array}{r}23.3 \\
(18.5,28.1)\end{array}$ & $\begin{array}{r}30.3 \\
(25.7,34.9)\end{array}$ & $\begin{array}{r}20.8 \\
(16.4,25.2)\end{array}$ & $\begin{array}{r}35.5 \\
(30.1,41.0)\end{array}$ & $\begin{array}{r}27.9 \\
(23.3,32.5)\end{array}$ & $\begin{array}{r}22.9 \\
(18.1,27.7)\end{array}$ & $\begin{array}{r}29.3 \\
(22.4,36.2)\end{array}$ \\
\hline 7 & Routine & $\begin{array}{r}31.6 \\
(30.2,33.1)\end{array}$ & $\begin{array}{r}35.1 \\
(29.0,41.2)\end{array}$ & $\begin{array}{r}45.1 \\
(40.5,49.8)\end{array}$ & $\begin{array}{r}31.7 \\
(27.5,35.9)\end{array}$ & $\begin{array}{r}20.8 \\
(17.1,24.5)\end{array}$ & $\begin{array}{r}40.0 \\
(35.2,44.7)\end{array}$ & $\begin{array}{r}18.9 \\
(15.3,22.5)\end{array}$ & $\begin{array}{r}34.2 \\
(29.2,39.2)\end{array}$ & $\begin{array}{r}28.3 \\
(24.3,32.2)\end{array}$ & $\begin{array}{r}22.4 \\
(18.5,26.4)\end{array}$ & $\begin{array}{r}36.8 \\
(30.5,43.0)\end{array}$ \\
\hline & All classes & $\begin{array}{r}19.0 \\
(18.6,19.4)\end{array}$ & $\begin{array}{r}23.7 \\
(21.4,25.9)\end{array}$ & $\begin{array}{r}26.9 \\
(25.5,28.3)\end{array}$ & $\begin{array}{r}18.3 \\
(17.0,19.7)\end{array}$ & $\begin{array}{r}15.5 \\
(14.1,16.8)\end{array}$ & $\begin{array}{r}23.6 \\
22.1,25.1)\end{array}$ & $\begin{array}{r}12.4 \\
(11.3,13.4)\end{array}$ & $\begin{array}{r}21.3 \\
(20.0,22.7)\end{array}$ & $\begin{array}{r}15.5 \\
(14.5,16.5)\end{array}$ & $\begin{array}{r}15.2 \\
(14.0,16.4)\end{array}$ & $\begin{array}{r}21.0 \\
(19.0,22.9)\end{array}$ \\
\hline & Ratio $7: 1$ & $\begin{array}{r}3.5 \\
(3.2,3.8)\end{array}$ & $\begin{array}{r}3.6 \\
(2.5,5.3)\end{array}$ & $\begin{array}{r}3.5 \\
(2.8,4.3)\end{array}$ & $\begin{array}{r}4.0 \\
(3.0,5.4)\end{array}$ & $\begin{array}{r}2.5 \\
(1.8,3.4)\end{array}$ & $\begin{array}{r}2.9 \\
(2.3,3.6)\end{array}$ & $\begin{array}{r}2.7 \\
(2.0,3.6)\end{array}$ & $\begin{array}{r}3.4 \\
(2.6,4.3)\end{array}$ & $\begin{array}{r}4.2 \\
(3.4,5.3)\end{array}$ & $\begin{array}{r}3.0 \\
(2.2,4.0)\end{array}$ & $\begin{array}{r}4.5 \\
(3.1,6.6)\end{array}$ \\
\hline
\end{tabular}

1 Directly age-standardised rates using the European standard population

2 95\% confidence intervals shown in parenthesis

3 Rates were not calculated where there were fewer than 10 deaths in a cell, denoted by $(:)$

4 Rates calculated from fewer than 20 deaths are shown in italics

Source: ONS 


\section{Table 9 Age-standardised mortality rates ${ }^{1,2,3,4}$ from alcohol-related causes of death, by NS-SEC classification and region, women aged 25-59, England and Wales, 2001-03}

Rate per 100,000

\begin{tabular}{|c|c|c|c|c|c|c|c|c|c|c|c|c|}
\hline \multirow{2}{*}{\multicolumn{2}{|c|}{ NS-SEC analytic classes }} & \multicolumn{11}{|c|}{ Regions of England and Wales } \\
\hline & & $\begin{array}{r}\text { England } \\
\text { and } \\
\text { Wales }\end{array}$ & $\begin{array}{r}\text { North } \\
\text { East }\end{array}$ & $\begin{array}{l}\text { North } \\
\text { West }\end{array}$ & $\begin{array}{l}\text { Yorshire } \\
\text { and The } \\
\text { Humber }\end{array}$ & $\begin{array}{r}\text { East } \\
\text { Midlands }\end{array}$ & $\begin{array}{r}\text { West } \\
\text { Midlands }\end{array}$ & $\begin{array}{l}\text { East of } \\
\text { England }\end{array}$ & London & $\begin{array}{r}\text { South } \\
\text { East }\end{array}$ & $\begin{array}{r}\text { South } \\
\text { West }\end{array}$ & Wales \\
\hline 1 & Higher managerial and professional & $\begin{array}{r}4.0 \\
(3.3,4.6)\end{array}$ & $\begin{array}{r}7.1 \\
(3.6,10.5)\end{array}$ & $\begin{array}{r}6.0 \\
(4.1,7.8)\end{array}$ & $\begin{array}{r}3.0 \\
(1.5,4.4)\end{array}$ & $\begin{array}{r}3.9 \\
(2.1,5.6)\end{array}$ & $\begin{array}{r}3.5 \\
(2.0,5.0)\end{array}$ & $\begin{array}{r}4.3 \\
(2.8,5.8)\end{array}$ & $\begin{array}{r}3.2 \\
(1.9,4.5)\end{array}$ & $\begin{array}{r}2.9 \\
(1.9,3.8)\end{array}$ & $\begin{array}{r}4.0 \\
(2.4,4.0)\end{array}$ & $\begin{array}{r}5.2 \\
(2.5,7.8)\end{array}$ \\
\hline 1.1 & Large employers and higher managerial & $\begin{array}{r}4.0 \\
(2.9,5.1)\end{array}$ & (:) & $\begin{array}{r}5.6 \\
(3.0,8.3)\end{array}$ & $(:)$ & $\begin{array}{r}4.4 \\
(2.0,6.9)\end{array}$ & $\begin{array}{r}5.1 \\
(2.6,7.5)\end{array}$ & $\begin{array}{r}3.7 \\
(1.7,5.6)\end{array}$ & $\begin{array}{r}3.6 \\
(1.5,5.6)\end{array}$ & $\begin{array}{r}2.2 \\
(1.1,3.3)\end{array}$ & $\begin{array}{r}4.3 \\
(2.0,6.6)\end{array}$ & $(:)$ \\
\hline 1.2 & Higher professional & $\begin{array}{r}3.9 \\
(3.1,4.7)\end{array}$ & $(:)$ & $\begin{array}{r}6.2 \\
(3.5,8.9)\end{array}$ & (:) & $(:)$ & $(:)$ & $\begin{array}{r}5.1 \\
(2.7,7.5)\end{array}$ & $\begin{array}{r}2.9 \\
(1.2,4.6)\end{array}$ & $\begin{array}{r}3.5 \\
(2.0,5.0)\end{array}$ & $\begin{array}{r}3.5 \\
(1.4,5.6)\end{array}$ & $(:)$ \\
\hline 2 & Lower managerial \& professional & $\begin{array}{r}6.5 \\
(5.9,7.1)\end{array}$ & $\begin{array}{r}11.1 \\
(8.3,13.8)\end{array}$ & $\begin{array}{r}9.0 \\
(7.4,10.6)\end{array}$ & $\begin{array}{r}6.6 \\
(5.1,8.1)\end{array}$ & $\begin{array}{r}5.9 \\
(4.3,7.4)\end{array}$ & $\begin{array}{r}6.4 \\
(4.9,7.9)\end{array}$ & $\begin{array}{r}4.2 \\
(3.1,5.2)\end{array}$ & $\begin{array}{r}5.7 \\
(4.5,6.9)\end{array}$ & $\begin{array}{r}6.2 \\
(5.1,7.2)\end{array}$ & $\begin{array}{r}5.5 \\
(4.1,6.8)\end{array}$ & $\begin{array}{r}6.8 \\
(4.8,8.8)\end{array}$ \\
\hline 3 & Intermediate & $\begin{array}{r}8.2 \\
(6.9,9.5)\end{array}$ & $\begin{array}{r}15.0 \\
(10.1,19.8)\end{array}$ & $\begin{array}{r}11.2 \\
(8.4,14.0)\end{array}$ & $\begin{array}{r}8.3 \\
(5.7,10.9)\end{array}$ & $\begin{array}{r}7.6 \\
(4.9,10.2)\end{array}$ & $\begin{array}{r}9.5 \\
(6.7,12.3)\end{array}$ & $\begin{array}{r}6.2 \\
(4.2,8.2)\end{array}$ & $\begin{array}{r}7.7 \\
(5.6,9.8)\end{array}$ & $\begin{array}{r}6.6 \\
(4.5,8.7)\end{array}$ & $\begin{array}{r}4.3 \\
(2.6,6.1)\end{array}$ & $\begin{array}{r}10.6 \\
(6.7,14.4)\end{array}$ \\
\hline 4 & Small employers \& own account workers & $\begin{array}{r}8.4 \\
(7.0,9.8)\end{array}$ & $\begin{array}{r}12.6 \\
(6.7,18.5)\end{array}$ & $\begin{array}{r}14.2 \\
(10.4,18.0)\end{array}$ & $\begin{array}{r}5.8 \\
(3.3,8.3)\end{array}$ & $\begin{array}{r}7.7 \\
(4.6,10.9)\end{array}$ & $\begin{array}{r}10.1 \\
(6.8,13.5)\end{array}$ & $\begin{array}{r}4.7 \\
(2.7,6.7)\end{array}$ & $\begin{array}{r}9.4 \\
(6.3,12.5)\end{array}$ & $\begin{array}{r}6.6 \\
(4.8,8.3)\end{array}$ & $\begin{array}{r}5.2 \\
(3.1,7.2)\end{array}$ & $\begin{array}{r}10.3 \\
(6.2,14.3)\end{array}$ \\
\hline 5 & Lower supervisory and technical & $\begin{array}{r}12.4 \\
(10.5,14.3)\end{array}$ & $\begin{array}{r}16.5 \\
(11.0,22.0)\end{array}$ & $\begin{array}{r}17.7 \\
(13.4,22.0)\end{array}$ & $\begin{array}{r}10.9 \\
(7.6,14.3)\end{array}$ & $\begin{array}{r}10.2 \\
(6.8,13.6)\end{array}$ & $\begin{array}{r}14.0 \\
(10.1,17.8)\end{array}$ & $\begin{array}{r}6.3 \\
(3.7,8.8)\end{array}$ & $\begin{array}{r}12.9 \\
(8.5,17.3)\end{array}$ & $\begin{array}{r}10.9 \\
(7.8,14.1)\end{array}$ & $\begin{array}{r}13.2 \\
(9.2,17.2)\end{array}$ & $\begin{array}{r}9.5 \\
(5.6,13.5)\end{array}$ \\
\hline 6 & Semi-routine & $\begin{array}{r}13.5 \\
(11.8,15.1)\end{array}$ & $\begin{array}{r}13.3 \\
(9.5,17.2)\end{array}$ & $\begin{array}{r}21.8 \\
(18.1,25.5)\end{array}$ & $\begin{array}{r}10.9 \\
(8.3,13.6)\end{array}$ & $\begin{array}{r}9.8 \\
(7.1,12.5)\end{array}$ & $\begin{array}{r}14.7 \\
(11.6,17.8)\end{array}$ & $\begin{array}{r}8.2 \\
(5.8,10.5)\end{array}$ & $\begin{array}{r}14.8 \\
(11.5,18.0)\end{array}$ & $\begin{array}{r}11.5 \\
(8.9,14.0)\end{array}$ & $\begin{array}{r}11.9 \\
(9.0,14.9)\end{array}$ & $\begin{array}{r}14.2 \\
(10.3,18.1)\end{array}$ \\
\hline 7 & Routine & $\begin{array}{r}22.4 \\
(19.7,25.2)\end{array}$ & $\begin{array}{r}21.2 \\
(14.8,27.6)\end{array}$ & $\begin{array}{r}35.3 \\
(29.1,41.6)\end{array}$ & $\begin{array}{r}17.7 \\
(13.2,22.3)\end{array}$ & $\begin{array}{r}15.3 \\
(11.0,19.6)\end{array}$ & $\begin{array}{r}25.3 \\
(19.7,30.9)\end{array}$ & $\begin{array}{r}16.7 \\
(11.7,21.7)\end{array}$ & $\begin{array}{r}23.4 \\
(17.3,29.5)\end{array}$ & $\begin{array}{r}18.8 \\
(13.8,23.9)\end{array}$ & $\begin{array}{r}17.7 \\
(12.5,23.0)\end{array}$ & $\begin{array}{r}25.9 \\
(18.6,33.2)\end{array}$ \\
\hline & All classes & $\begin{array}{r}9.0 \\
(8.7,9.4)\end{array}$ & $\begin{array}{r}13.2 \\
(11.5,14.9)\end{array}$ & $\begin{array}{r}14.1 \\
(13.0,15.2)\end{array}$ & $\begin{array}{r}8.4 \\
(7.4,9.3)\end{array}$ & $\begin{array}{r}7.8 \\
(6.8,8.8)\end{array}$ & $\begin{array}{r}10.3 \\
(9.2,11.3)\end{array}$ & $\begin{array}{r}5.9 \\
(5.1,6.6)\end{array}$ & $\begin{array}{r}8.3 \\
(7.4,9.2)\end{array}$ & $\begin{array}{r}7.0 \\
(6.3,7.7)\end{array}$ & $\begin{array}{r}7.2 \\
(6.4,8.1)\end{array}$ & $\begin{array}{r}10.5 \\
(9.1,11.9)\end{array}$ \\
\hline & Ratio 7:1 & $\begin{array}{r}5.7 \\
(4.7,6.9)\end{array}$ & $\begin{array}{r}3.0 \\
(1.9,4.8)\end{array}$ & $\begin{array}{r}5.9 \\
(4.3,8.2)\end{array}$ & $\begin{array}{r}6.0 \\
(3.8,9.4)\end{array}$ & $\begin{array}{r}4.0 \\
(2.5,6.2)\end{array}$ & $\begin{array}{r}7.2 \\
(4.8,10.8)\end{array}$ & $\begin{array}{r}3.8 \\
(2.6,5.7)\end{array}$ & $\begin{array}{r}7.4 \\
(4.9,11.2)\end{array}$ & $\begin{array}{r}6.6 \\
(4.5,9.5)\end{array}$ & $\begin{array}{r}4.5 \\
(2.9,6.8)\end{array}$ & $\begin{array}{r}5.0 \\
(3.1,8.1)\end{array}$ \\
\hline
\end{tabular}

1 Directly age-standardised rates using the European standard population

2 95\% confidence intervals shown in parenthesis

3 Rates were not calculated where there were fewer than 10 deaths in a cell, denoted by $(:)$

4 Rates calculated from fewer than 20 deaths are shown in italics

Source: ONS

\section{Age-standardised mortality rates by NS-SEC - Men}

A large socio-economic gradient in mortality rates was found between men in Higher managerial and professional occupations (Class 1 ) and men in Routine occupations (Class 7), with the latter 3.5 times as likely to die from alcohol-related causes as the former in the period examined (Table 8). The highest alcohol-related mortality rate of all classes occurred for Routine workers, at 31.6 per 100,000 .

Men in each of the three Routine and Manual classes (Classes 5, 6 and 7) had statistically significantly higher rates of alcohol-related mortality than all men in England and Wales. Mortality rates of Routine workers (Class 7) were approximately 64 per cent higher than those of all men in England and Wales. By contrast, men assigned to each of the Managerial and professional classes (Classes 1.1, 1.2 and 2) as well as Small employers and own account workers (Class 4) had statistically significantly lower rates of alcohol-related mortality than all men in England and Wales. Higher managers and professionals (Class 1 ) had a mortality rate approximately half that of all men in England and Wales. 
There was a marked statistical difference in alcohol-related mortality between the two subgroups of the Higher managerial and professional occupations (Classes 1.1 and 1.2), and between the Lower supervisory and technical class (Class 5 ) and the Semi-routine class (Class 6), as illustrated in Figure 3. Men in Intermediate occupations (NS-SEC class 3 ) had similar mortality rates to those of Small employers and own account workers (NS-SEC class 4), at around 17 per 100,000. This was a result of the mortality rates for men in Intermediate occupations being lower than those of Small employers and own account workers at younger ages and higher at older ages (see Figure 1).

\section{Age-standardised mortality rates by NS-SEC - Women}

The mortality rates from alcohol-related causes were consistently lower for women than men across the NS-SEC analytic classes, as seen in Figure 3 . The mortality rate of women in Routine occupations was the highest of all classes, at 22.4 per 100,000, and was similar to that of men in the Lower supervisory and technical class (Class 5).

The socio-economic inequalities in alcohol-related mortality for women were greater than those seen for men, with women in the Routine class having a rate of alcohol-related mortality about six times greater than women in Higher managerial and professional occupations (Table 9).

Unlike for men, the mortality rates experienced by women in Lower supervisory and technical class (Class 5) were similar to those for women in the Semi-routine class (Class 6). Women in Routine occupations (Class 7 ) had a significantly raised mortality from alcohol-related causes compared to women in Semi-routine occupations (Class 6). Also, there were no statistically significant differences between the mortality of women in Higher and lower managerial and professional occupations (Classes 1.1 and 1.2).

\section{Wales, England and the regions of England: age-standardised mortality rates by NS- SEC}

The age-standardised alcohol-related mortality rates for men aged 25-64 and for women aged 2559 by NS-SEC class for England and Wales, the regions of England, and Wales are shown in Tables 8 and 9 and illustrated in Figure 4.

The socio-economic gradients or ratios of mortality rates of men (or women) in Routine occupations (Class 7 ) to that of men (or women) in Higher managerial and professional occupations (Class 1), are also presented in Tables 8 and 9 and illustrated individually in Figures 5 and 6.

Results for England on the other hand will not be covered by this analysis due to the close proximity to the England and Wales results; however data on rates for England can be found in supplementary tables published on the ONS website at: www.statistics.gov.uk/hsq/downloads/hsq50art1.xls 
Figure 4 Age-standardised mortality rates ${ }^{1}$ from alcohol-related causes of death by NS-SEC, men aged 25-64 and women aged 25-59, England and Wales, the regions of England and Wales, 2001-03
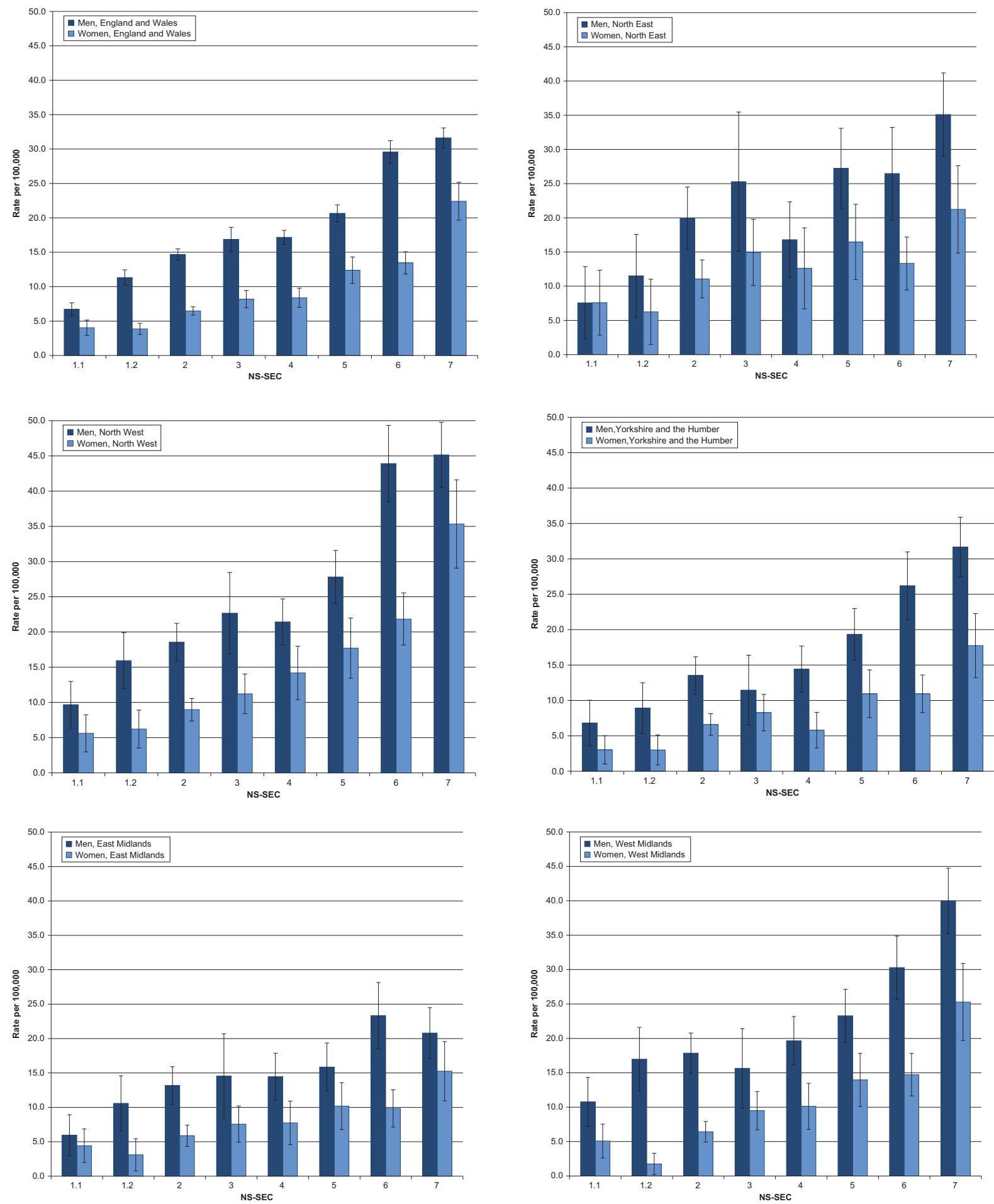

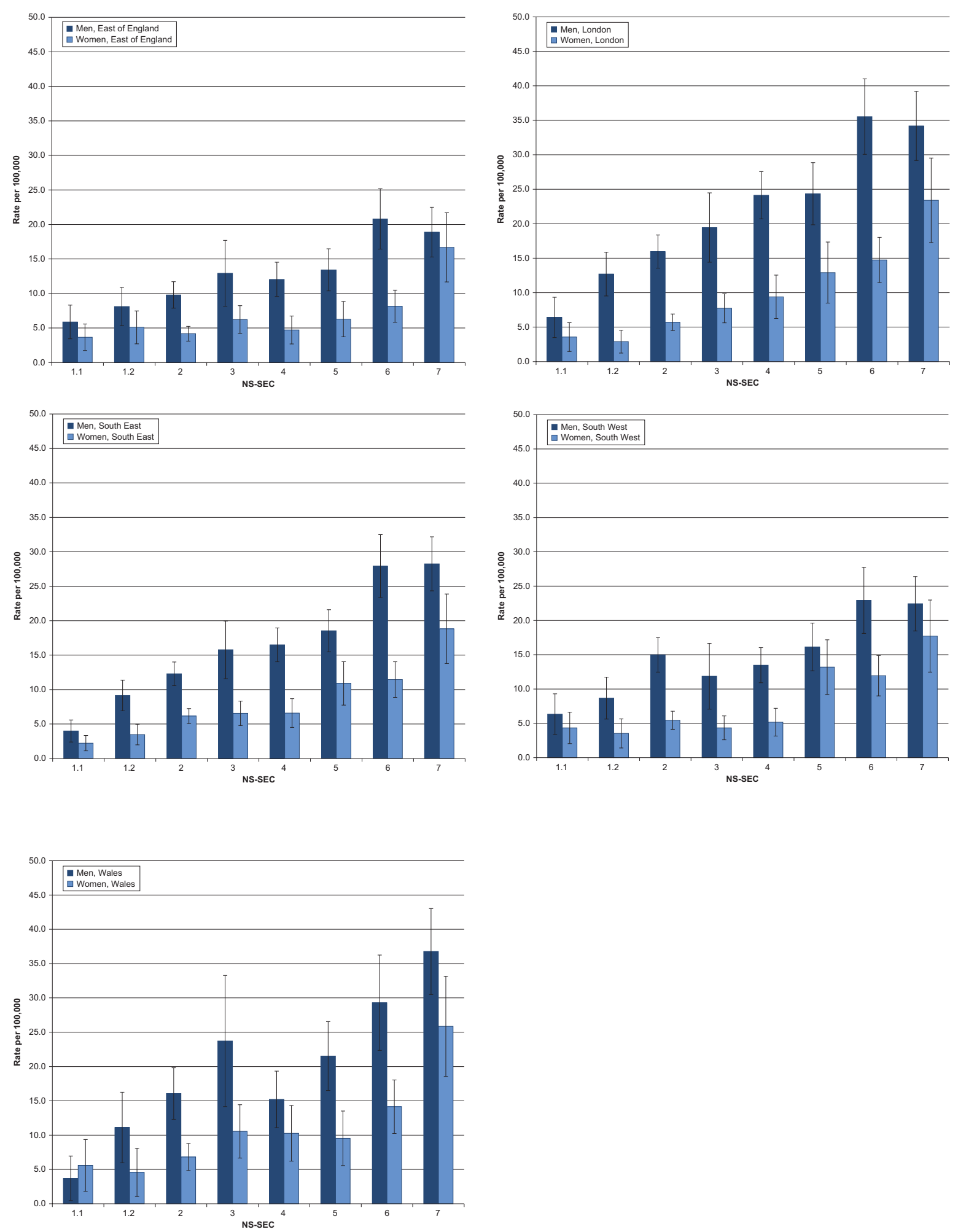

1 Directly age-standardised rates using the European standard population

Source: ONS 


\section{Figure 5 Socio-economic gradients ${ }^{1}$ in mortality for alcohol-related causes of death, for men aged 25-64, in England and Wales, the regions of England and Wales, 2001-03}

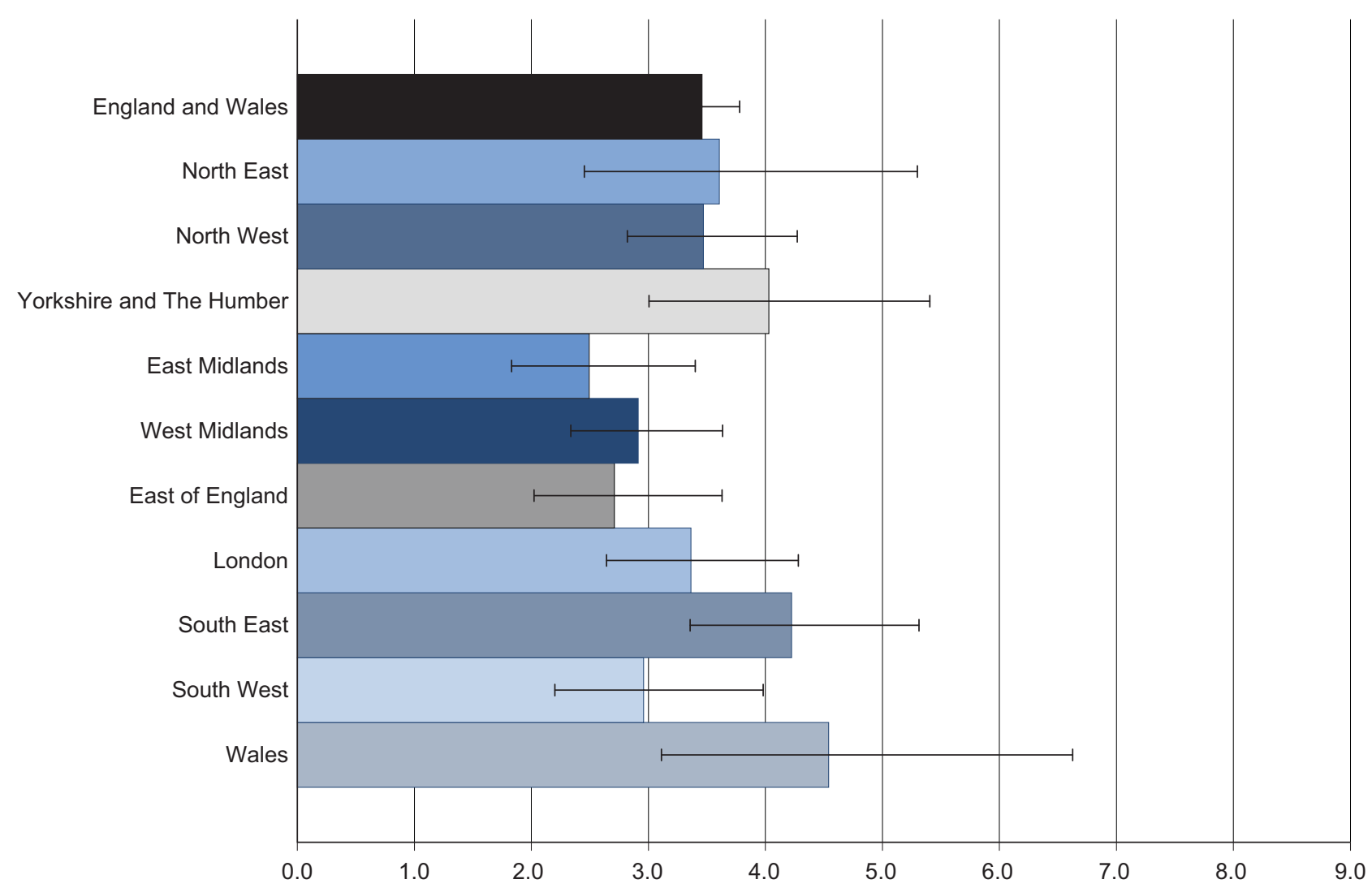

1 Ratio of the mortality rate of men in NS-SEC class 7 to the mortality rate of men in NS-SEC class 1

Source: ONS 


\section{Figure 6 Socio-economic gradients ${ }^{1}$ in mortality for alcohol-related causes of death, for women aged 25-59, in England and Wales, the regions of England and Wales, 2001-03}

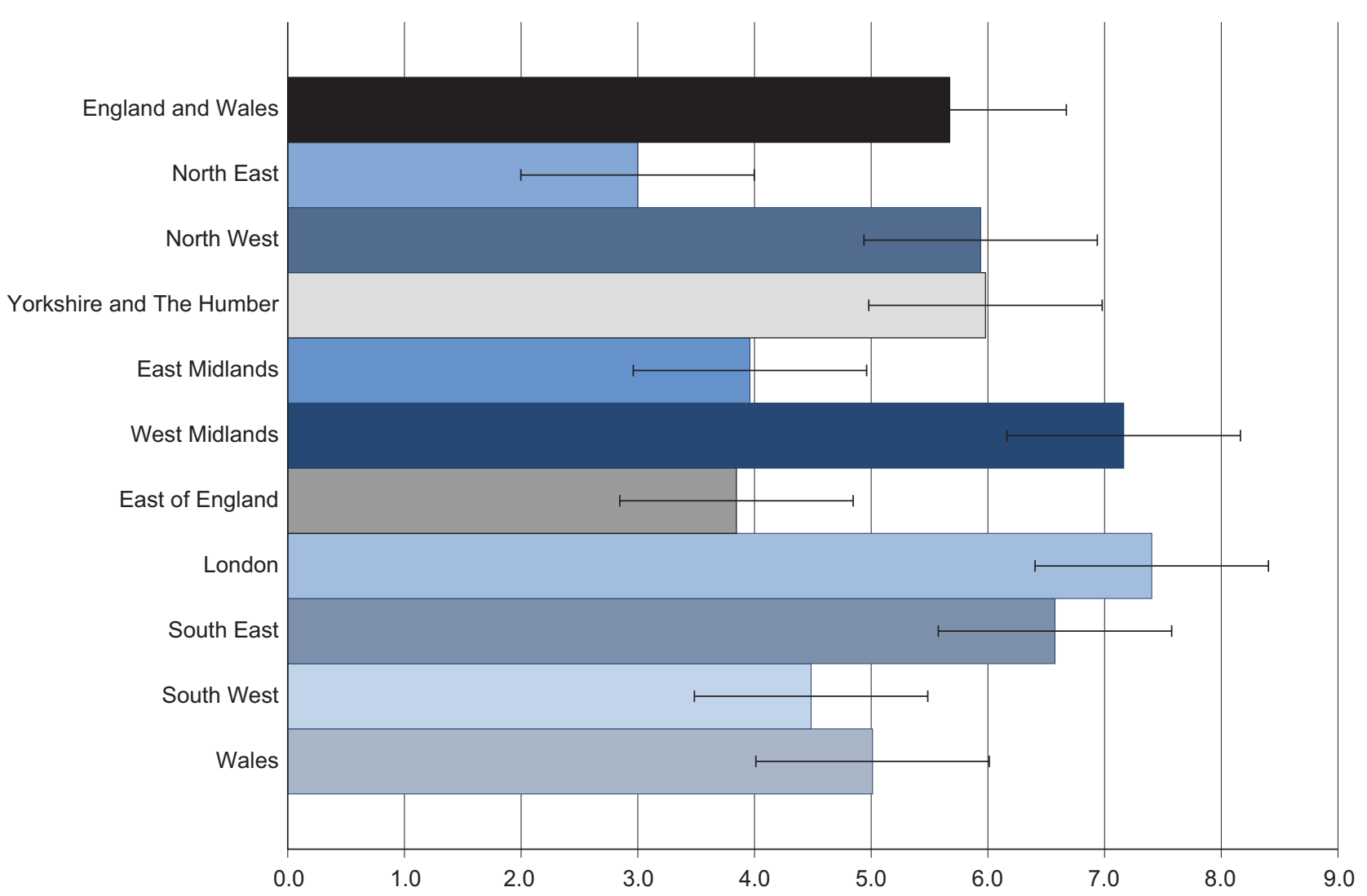

1 Ratio of the mortality rate of women in NS-SEC class 7 to the mortality rate of women in NS-SEC class 1

Source: ONS

\section{Age-standardised mortality rates by NS-SEC - Men}

The highest mortality rate for all occupied classes was observed in the North West (26.9 per 100,000), followed by the North East (23.7 per 100,000), the West Midlands (23.6 per 100,000) and London (21.3 per 100,000) (Table 8 ). These regions all had significantly higher mortality rates for all occupied classes than England and Wales as a whole (19.0 per 100,000).

In the North West, the overall higher mortality rate compared to England and Wales as a whole was characterised by raised rates in each individual class. The death rate for men in Routine occupations (Class 7) in the North West was 45.1 per 100,000, which represented the highest rate in this study and was 43 per cent higher than for Routine workers in England and Wales as a whole (Figure 4 and Table 8).

In the North East, the West Midlands and London, the mortality rates were greater than for England and Wales in most classes (Table 8). A statistically significant difference in rates occurred 
for the Small employers and own account workers class (Class 4) in London, which had a 41 per cent higher rate of alcohol-related mortality than in England and Wales (Table 8).

The lowest mortality rate for all occupied classes together occurred in the East of England, with a value $(12.4$ per 100,000$)$ half that observed in the North West. The second lowest mortality rate for all occupied classes was in the South West (15.2 per 100,000), followed by the East Midlands and the South East (both 15.5 per 100,000).

The mortality rates of men in the South West, the East Midlands and the South East were lower than in England and Wales in most classes. The lowest mortality rate for men in this study occurred for Large employers and higher managers (Class 1.1) in the South East and Wales (approximately 4 per 100,000).

The socio-economic differences in alcohol-related mortality between Routine workers (Class 7) and Higher managers and professionals (Class 1) ranged between 2.5 (in the East Midlands) and 4.5 (in Wales) (Figure 5).

\section{Age-standardised mortality rates by NS-SEC - Women}

The regions with the highest mortality rates for occupied classes as a whole were the same for women as for men (Figure 4). The mortality rates in all occupied classes in the North-West (14.1 per 100,000) and in the North East (13.2 per 100,000) were significantly higher than in England and Wales as a whole (Table 9).

The mortality rates of women were higher in the North West than in England and Wales in each individual class. Women in Routine occupations (Class 7) in the North West had a rate of alcoholrelated mortality (35.3 per 100,000) of a similar order of magnitude to men in Routine occupations (Class 7 ) in England and Wales as a whole (31.6 per 100,000).

Women in the North East had elevated mortality rates compared to England and Wales in most classes except in Semi-routine and Routine classes (Classes 6 and 7).

The lowest mortality rate for all women in occupied classes occurred in the East of England with a value (5.9 per 100,000) around 40 per cent lower than the one observed in the North West (14.1 per 100,000). The lowest mortality rate for women in this study occurred for Large employers and higher managers (Class 1.1) in the South East (approximately 2 per 100,000).

The socio-economic differences in alcohol-related mortality between Higher managers and professionals (Class 1 ) and Routine workers (Class 7 ) were consistently greater for women than for men across regions (Tables 8 and 9), except in the North East. Alcohol-related mortality rates were between five and 7.5 times higher for routine workers compared to Higher managers and professionals in the North West, Yorkshire and The Humber, West Midlands, London, South East and Wales. The highest relative difference between the Routine occupations and the Higher managerial and professional occupations (Class 1) was a factor of 7.4 in London. 


\section{Discussion}

This analysis gives the first detailed breakdown of alcohol-related deaths by NS-SEC classes, age, gender and region. The results support both national and international literature reporting significant inequalities in alcohol-related mortality across socio-economic classes by age, gender and area (Romeri et al., 2007; Harrison and Gardiner, 1999; Makela et al., 1997).

For men, the socio-economic gradients presented in this article show that workers in Routine occupations (Class 7 ) had a rate of alcohol-related mortality about 3.5 times greater than those in Higher managerial and professional occupations (Class 1), which is higher than the gradients seen in male mortality from all circulatory diseases, all cancers and accidents (White et al., 2008). These results are consistent with studies of male inequalities in alcohol-related mortality made in the last 20 years.

Breakwell et al., 2007, using the Carstairs and Morris index of deprivation, found that there was a five-fold alcohol-related mortality ratio between the most and least deprived twentieths of the population of men (in the UK). Analysis of mortality by occupation (Romeri et al., 2007) found that men tended to have higher Standardised Mortality Ratios (SMRs) for alcohol-related diseases in occupations associated with the Routine NS-SEC grouping. An earlier study using data from 198894 (Harrison and Gardiner, 1999) found that men in Social Class V (Unskilled Manual) had an SMR of 267 compared with 59.8 in Social Class I (Professional). By contrast, earlier data suggest that the social gradient in alcohol-related mortality was reversed in the middle of the 20th century. In 1951 liver disease mortality was higher for those in social class I, with a standard mortality ratio of 128 compared with 111 for social class V, and 63 for social class IV (OPCS, 1951).

For women, the results in the current study suggest that workers in Routine occupations had 5.7 times the mortality rate from alcohol-related causes than those working in Higher managerial and professional occupations, higher than the socio-economic gradients in mortality seen for women from cerebrovascular diseases and all cancers (Langford et al., 2009). This is also substantially greater than the inequalities observed for men.

Previous literature concerning social variations in alcohol-related mortality for women suggests a possible change in the pattern of socio-economic inequalities in the last 20 years. Breakwell et al., 2007, found that women in the most deprived five per cent of areas had alcohol-related mortality more than three times higher than those in the least deprived areas, a finding supporting the estimates of the current study. Erskine et al. (2010) also found a clear association between alcohol-related mortality and deprivation for women.

Evidence from the 1990s suggests that the social gradient in mortality for women was reversed at that time. The work of Harrison and Gardiner (1999) found that women in employment in Social Class V had a Standardised Mortality Ratio (SMR) in Social Class V of 68.4 compared to 149.6 in Social Class I. However, in that study, younger women in the manual classes were more likely to die from alcohol-related causes, but for older women it was those in the professional class who suffered elevated mortality. It is possible that the 'combined' NS-SEC classification used in the current analysis tended to increase the socio-economic gradient for women relative to earlier studies which referred only to working women and used the woman's own occupation. 
For both men and women, age-specific mortality rates presented in this article show that the risk of alcohol-related mortality increased steeply from younger ages before either reaching a plateau or beginning to decrease in most NS-SEC classes, except for the most advantaged. The peak in alcohol-related mortality for Routine workers (Class 7) occurred at ages 50-54 for men compared to 45-49 for women. By contrast, for Managers and Professionals (Classes 1 and 2), the greatest alcohol-related mortality was observed in the oldest age group studied (55-59 for women and 6064 for men), as it does for most non-external causes of death.

Mortality data (ONS, 2011) suggest that, despite advances in medical science, people in England and Wales are increasingly dying from alcohol-related causes at earlier ages. In 1991 there were 3,415 deaths directly attributable to alcohol. In men, 42 per cent of deaths were under the age of 55, and in women, 34 per cent. By 2009 the aggregate had grown to 7,099 deaths with 47 per cent under the age of 55 for men and 44 per cent for women. Baker and Rooney (2003) also showed that the increase in alcohol-related mortality rates between 1979 and 2000 was particularly large for men and women aged 25-44, indicating a faster rise in alcohol-related mortality at younger ages, across both genders over a period of 20 years.

Studies encompassing the last 20 years suggest that inequalities in alcohol-related mortality are wider at younger ages, at least for men. Erskine et al. (2010) suggested that for both men and women, the greatest alcohol-related mortality rate ratios for the most socio-economically deprived quintile of the population of England and Wales relative to the least deprived in 1999-2003, occurred at the ages 25-44 years. Harrison and Gardiner (1999) using data from 1988-92 found that men aged 25-39 in the unskilled Manual class (Social Class V) were 10-20 times more likely to die from alcohol-related causes than those in the Professional Social Class (Social Class I), whereas men aged 55-64 in Social Class $V$ were only about 2.5-4 times more likely to die than their professional counterparts. However, the study of Harrison and Gardiner also suggested reverse inequalities for women aged 55-64.

The underlying reasons for the marked socio-economic gradient in alcohol-related deaths for both men and women are not clear. There are co-factors for the development of alcohol-related liver disease, of which perhaps the best documented is obesity (Hart et al., 2010; Liu et al., 2010). It has also been suggested that the gradient may be related to social drift, the idea that heavy drinkers tend to move down the social class scale. However, there is very limited evidence for such 'social selection' effects on mortality for other causes of death (Blane et al.,1999).

The key risk factor for alcohol-related death is alcohol intake and the obvious conclusion would be that men and women in Routine occupations (Class 7) drink considerably more than those in Higher managerial and professional occupations (Class 1). However, repeated population-based sampling surveys have suggested an inverse relationship between alcohol consumption and socioeconomic class. The latest results of the General Lifestyle Survey (GLF) found that in 2009 in Large employer and higher managerial households, 25 per cent of men exceeded eight units and 18 per cent of women exceeded six units on at least one day in the week before interview, compared to 19 per cent of men and 9 per cent of women in Routine households (GLF, 2009). GLF data also suggested that the average alcohol weekly consumption in 2009 was 15.7 units in Large employers and higher managerial households compared to 10.7 units in Routine households (GLF, 2009). 
Obtaining reliable information about drinking behaviour is known to be extremely difficult (Goddard et al., 2001) and surveys such as the GLF consistently record lower levels of alcohol consumption than estimates based on alcohol sales. People may consciously or unconsciously underestimate the amount of alcohol they have consumed; this is a particular risk when the drinking occurs at home because the quantities consumed are not measured and are likely to be larger than those dispensed in licensed premises.

There is a particular problem obtaining accurate data for individuals consuming alcohol at levels outside social norms and for whom by definition the risk of alcohol-related death would be greater than the general population. It has been suggested that the addition of an established alcohol question set such as the $\mathrm{WHO}$ audit questionnaire to surveys might improve data quality (Saunders et al., 1993).

It has been suggested that drinking patterns vary across socio-economic classes. The Marmot review (2010), commissioned by the Department of Health, acknowledged an inverse gradient for alcohol consumption, but highlighted that people with lower socio-economic status who consumed alcohol were more likely to have problematic drinking patterns and dependence. A study using data from the 1958 British Cohort Study showed that although men in unskilled manual social groups had higher levels of non-drinking, they were also more likely to binge drink (defined as drinking more than ten units of alcohol on one occasion) (Jefferis et al., 2007).

It is likely that both aggregate deaths and the socio-economic differences in mortality rates observed at younger ages are largely determined by drinking patterns and habits acquired at a young age. Baker and Rooney (2003) showed that both sexes experienced a birth cohort effect between 1979 and 2000, whereby almost every successive five-year birth cohort had a higher alcohol-related mortality rate than the previous one at a given age. This tendency to carry drinking behaviour through life could partly explain some of the differences observed at different time periods. For example, the apparent inconsistency between the social gradients in mortality for women seen in the current study and that of Harrison and Gardiner (1999) might be the effect of a change over time in the drinking behaviour among young women in less advantaged classes, which was beginning to affect middle-aged women by 2001-03.

Despite the lack of clear evidence on class differences in alcohol consumption, it appears that trends in alcohol pricing have an effect on the socio-economic gradients seen in alcohol-related mortality. The recent modelling of the effect of the price of alcohol on rates of death and morbidity commissioned by the Department of Health, suggests the close links between price, consumption and ill health (Brennan et al., 2009). The House of Commons, Alcohol, Health Committee report showed that the rate of duty on spirits per litre of pure alcohol in 1947 would have cost almost 60 per cent of the weekly average manual earnings of a man, but by 2002 the figure was less than 10 per cent (Health Select Committee, 2010). Information from the NHS Information Centre suggests that alcohol was 75 per cent more affordable in 2008 than in 1980 (NHS Health and Social Care Information Centre, 2009). It appears that as the relative price of alcohol has fallen, so the associated social gradient of deaths has reversed. The fact that alcohol has become more affordable might therefore affect different socio-economic groups differently.

The geographic variations in socio-economic differences found in this study were similar to those found for other causes (Siegler et al., 2008) In particular alcohol-related mortality rates for both 
sexes in the North West were significantly higher than the corresponding rates for England and Wales across several of the NS-SEC classes. The socio-economic gradients were higher for women compared to men in all areas with the exception of the North East where the gradient for women was 3.0 compared with 3.6 for men. The largest inequalities for men were observed in Wales (4.5), and for women were in London (7.4). In general, though, regional socio-economic gradients were less correlated with the general level of mortality than for other causes (Siegler et al., 2008). For example, the socio-economic gradient in the North East was less than that in the South East, while aggregate deaths in the North East were greater.

While the socio-economic differences are clear in terms of mortality rates and age of death, the causes of these differences remain complex and can evolve rapidly as society changes.

\section{Limitations of the analysis}

The mortality rates reported here are from the causes which were agreed to be directly attributable to alcohol as defined by ONS for the purposes of comparison of trends (ONS Discussion paper, 2006). The figures do not take into account all deaths with a mention of alcohol and they do not reflect deaths in which alcohol was an attributable factor such as cancer of the upper gastrointestinal tract or breast. Deaths from accidents and violence caused by drinking are also excluded.

The commonest causes of death from binge drinking are intentional self-harm and road traffic accidents which occur most commonly in young people. According to analyses performed by the North West Public Health Observatory (Jones et al., 2008) 22-26 per cent of deaths in young males, and 19-23 per cent of deaths in young females, in the 16-44 age band in England are alcohol-related. The majority of these deaths are not recorded as being due to alcohol.

Recent research has suggested that alcohol-related liver disease may be under-reported on the death certificate. A recent audit - by one of the authors of this report - of 477 deceased patients with alcohol-related liver disease found that 22 per cent had no mention of liver disease or alcohol on the death certificate (Sheron N., unpublished data). Conversely, the inclusion of all deaths from cirrhosis and chronic liver fibrosis, except those from primary biliary cirrhosis, in the alcohol-related definition may over estimate the number of deaths to some extent. In 2004, the Department of Health estimated there were 4,000-4,100 acute alcohol-related deaths and 11,300-17,900 deaths due to regular heavy drinking in England (Prime Minister's Strategy Unit, 2004).

A limitation when considering alcohol-related mortality by NS-SEC is the risk that people who misuse alcohol may be less likely to be in employment, therefore leading to a high proportion of alcohol-related deaths being excluded from this analysis. Results from this study indicate that 15 per cent of male deaths and 9 per cent of female deaths were excluded as their NS-SEC category was the 'non-occupied' group. Previous studies looking at the alcohol-related mortality rates by occupation also showed that there was a higher proportion of deaths with no occupation recorded when the underlying cause of death was alcohol-related compared with all registered deaths (Romeri et al., 2007). Many people who were not in employment at the time of death were assigned to an NS-SEC category via a previous occupation. Nevertheless, a residual group remained impossible to classify by occupation and therefore by NS-SEC, and some of these may be among the most deprived. 
In previous studies of health inequalities of adult mortality by socio-economic group, ONS has typically focused on either men or women. In this analysis, both men and women were considered and this poses some restriction in comparing their results. It was necessary to restrict the analysis for women to those aged 25-59, while for men it was for ages 25-64, since recording of occupation at census above this age is sparse (and non-existent above the age of 74).

Due to the good recording of husbands' occupations for women at death and the severe under recording of wife's occupation reported earlier, in this analysis a 'combined' classification approach was employed for women but not for men. The death registers during the period of the study did not recognise civil partnerships and thus women in such partnerships have been treated as single for the purpose of assigning an NS-SEC class. The same definition was used to obtain the census population so the results presented are consistent. However there is an argument for treating women and men in partnerships in the same way as if they were married.

In some of the findings presented, the number of underlying deaths was small, especially at regional level, leading to wide confidence intervals. This may lead to a degree of uncertainty in the interpretation of the resultant socio-economic gradients. However, the authors have been careful not to draw stronger conclusions than seem merited by the estimates.

Selective migration between regions may lead to bias since deaths are assigned to the region of usual residence at death, whereas the denominator is calculated using census data allocated to regions according to the workforce in 2001.

The study period was restricted to the period 2001-03 since the numerator and denominator must relate to the same period and it has only been possible to obtain population denominators classified by age, sex and socio-economic class, at the time of the decennial Census in 2001. In addition, the NS-SEC classification was introduced into official statistics only in 2001. For these reasons, the numerators and denominators were restricted to the three years beginning with that of the 2001 Census. The census figures were adjusted to reflect ONS estimated mid-year populations for 2001, 2002 and 2003. A series of analyses by NS-SEC (for example, males, males by cause, by region) were undertaken using the same denominators throughout (Langford et al., 2009; Langford and Johnson, 2009; Siegler et al., 2008; White et al., 2008; White et al., 2007).

\section{Conclusions}

This article presents the first analysis of alcohol-related mortality by NS-SEC for men and women of working age, using the National Statistics definition of alcohol-related mortality. The general socio-economic pattern of alcohol-related mortality found was consistent with previous research.

Substantial socio-economic gradients in alcohol-related mortality rates were observed both across England and Wales as a whole, and within the English regions and Wales. Men in Routine occupations had alcohol-related mortality rates 3.5 times higher than Higher managers and professionals, and for women the corresponding multiple was even higher, at 5.7. These inequalities in mortality were wider at younger ages. This was accounted for by the fact that alcohol-related mortality rates increased rapidly and peaked at younger ages in the Routine class (Class 7) whereas for Higher managers and professionals (Class 1 ) the rates increased steadily with age. 
While these socio-economic gradients were present for both men and women, analysis of agespecific mortality rates suggested that the age at which mortality peaked differed between genders. The highest mortality rate among classes and age groups was observed in the Routine class for both men and women, but at ages 50-54 for men (52.2 per 100,000) compared with ages 45-49 for women (42.0 per 100,000).

There were further gender differences in the socio-economic patterns in alcohol-related mortality. Both men and women in the less advantaged classes (Classes 5, 6 and 7) had significantly higher rates of alcohol-related mortality than in England and Wales as a whole. However, women in Routine occupations (Class 7) had significantly higher mortality rates than women in Semi-routine occupations (Class 6), while rates for men in these classes showed no significant difference. The mortality rates of men in Semi-routine occupations (Class 6) were significantly higher than those in Lower supervisory and technical occupations (Class 5), and this was not the case for women.

Within England, the North West had the highest overall rates of alcohol-related mortality with particularly high rates in the Routine class for both sexes (45.1 per 100,000 population for men and 35.3 per 100,000 for women). The socio-economic gradients in mortality were higher for women than for men in all regions with the exception of the North East. In general, there was no association between the socio-economic gradients and the level of mortality across regions. For example, the North East had the lowest socio-economic gradient in mortality of all regions for women, despite having the second highest mortality rate of all regions.

The strong socio-economic gradient in alcohol-related deaths cannot be readily explained in terms of differences in the amounts of alcohol consumed. Further research is needed to identify what additional factors may determine the higher mortality risk found in less advantaged NS-SEC classes. However, historical data are consistent with the hypothesis that as the relative price of alcohol has fallen, so the social gradient has increased. This article does not attempt to address the economic issues involved in alcohol policy, but provides information on socio-economic patterns which may be valuable to assist in the development of public health policies to reduce alcohol-related harm. 


\section{References}

Baker A and Rooney C (2003). Recent trends in alcohol-related mortality, and the impact of ICD-10 on the monitoring of these deaths in England and Wales. Health Statistics Quarterly 17, 5-15. Available on the Office for National Statistics website at: www.statistics.gov.uk/statbase/product.asp?vlnk $=6725$

Blane D, Harding S, Rosato M (1999). Does social mobility affect the size of the socio-economic mortality differential?: evidence from the Office for National Statistics Longitudinal Study. Journal of the Royal Statistical Society, 162 part 1, 59-70.

Breakwell C, Baker A, Griffiths C, Jackson G, Fegan G, and Marshall D (2007).Trends and geographical variations in alcohol-related deaths in the United Kingdom, 1991-2004, Health Statistics Quarterly 33, 6-24. Available on the Office for National Statistics website at: www.statistics.gov.uk/statbase/product.asp?vlnk $=6725$

Brennan A, Purshouse R, Taylor K, Rafia R, Booth A, O'Reilly D et al. (2008). Independent review of the effects of alcohol pricing and promotion. Part B: Modelling the potential Impact of pricing and promotion policies for alcohol in England. Results from the Sheffield Alcohol Policy Model version 2008. School of Health and Related Research at the University of Sheffield (SCHARR). Available at: www.bvsde.paho.org/bvsacd/cd68/AlcoholPartB.pdf

British Beer and Pub Association and HM Revenue and Customs (2009). BBPA Statistical Handbook 2009.

Cabinet Office (2010). Applying behavioural insight to health. Discussion Paper.

Department of Health (2010) White Paper: Healthy lives, Healthy people: Our strategy for Public Health in England.

Erikson R (1984). 'Social class of men, women and families', Sociology, 18, 500-14.

Erskine S, Maheswaran R, Pearson T, Gleeson D (2010). Socioeconomic deprivation, Urban-rural location and alcohol-related mortality in England and Wales, BMC Public Health, 2-8.

General Household Survey (GHS) and General Lifestyle Survey (GLF), Office for National Statistics, available at:

www.statistics.gov.uk/StatBase/Product.asp?vlnk=5756\&Pos=3\&ColRank=2\&Rank=416

Goddard E (2001). Obtaining information about drinking though surveys of the general population. National Statistics Methodology; Series No.24, ONS: London.

Harrison L and Gardiner E (1999). Do the rich really die young? Alcohol-related mortality and social class in Great Britain, 1988-94, Addiction, volume 94, number 12, p: 1871-1880.

Hart CL, Batty GD,Morrison DS, Mitchell RJ, Smith GD. Obesity, overweight and liver disease in the Midspan prospective cohort studies. Int J Obes (Lond) 2010; 34(6):1051-1059. 
Health Survey for England (2011). Available at: www.ic.nhs.uk/statistics-and-datacollections/health-and-lifestyles-related-surveys/health-survey-for-england

House of Commons Health Committee (2010). Alcohol-First Report of Session 2009-10, Volume I:, Report Together with Formal Minutes.

Jefferis BJ, Manor O, Power C (2007). Social gradients in binge drinking and abstaining: trends in a cohort of British adults. J Epidemiol Community Health; 61:150-153.

Jones L, Bellis MA, Dedman D, Sumnall H, Tocque K (2008). Alcohol attributable fractions for England; alcohol attributable mortality and hospital admissions. North-West Public Health Observatory and Dept of Health. Available at: www.nwph.net/nwpho/publications/alcoholattributablefractions.pdf

Langford A and Johnson B (2009). Social inequalities in adult female mortality by the National Statistics Socio-economic Classification, England and Wales, 2001-03. Health Statistics Quarterly 42, 6-21. Available on the Office for National Statistics website at: www.statistics.gov.uk/statbase/product.asp?vlnk $=6725$

Langford A, Johnson B, Al-Hamad A (2009). Social inequalities in female mortality by region and by selected causes of death, England and Wales, 2001-03. Health Statistics Quarterly 44, 7-26. Available on the Office for National Statistics website at: www.statistics.gov.uk/statbase/product.asp?vlnk $=6725$

Liu B, Balkwill A, Reeves G, Beral V (2010). Body mass index and risk of liver cirrhosis in middle aged UK women: prospective study. British Medical Journal ; 340:c912.

Makela P, Valkonen T and Martelin T (1997). Contribution of deaths related to alcohol use to socioeconomic variation in mortality: register based follow up study, British Medical Journal; 315:211-216, 1-8.

Marmot Review (2010). Strategic Review of Health Inequalities in England post 2010: Fair Society, Healthy Lives, London.

NHS Health and Social Care Information Centre (2010). Smoking, drinking and drug use among young people in England in 2009.

NHS Health and Social Care Information Centre (2010). Adult Psychiatric Morbidity in England, 2007; Chapter 9: Alcohol misuse and dependence.

NHS Health and Social Care Information Centre (2009). Statistics on Alcohol: England, 2009.

Office for National Statistics (2011). Alcohol Related Deaths in the United Kingdom, 1991-2009. Available on the Office for National Statistics website at:

www.statistics.gov.uk/StatBase/Product.asp?vlnk=14496

Office for National Statistics Discussion paper (2006), Defining alcohol-related deaths. Available on the Office for National Statistics website:

www.statistics.gov.uk/downloads/theme health/Defining alcohol-related deaths.pdf 
Office of Population Censuses and Surveys (OPCS) (1951) The Registrar General's Decennial Supplement for England and Wales, 1951, Part 1, p39

Prime Minister's Strategy Unit (2004) . Alcohol Harm Reduction Strategy for England. Interim Analytical Report.

Romeri E, Baker A and Griffiths C (2007). Alcohol-related deaths by occupation, England and Wales, 2001-05. Health Statistics Quarterly 35, 6-12. Available on the Office for National Statistics website at: www.statistics.gov.uk/statbase/product.asp?vlnk $=6725$

Rose D and Pevalin D (eds) (2003). A Researcher's Guide to the National Statistics Socioeconomic Classification, Sage: London.

Saunders JB, Aasland OG, Babor TF, de la Fuente JR, Grant M. (1993). Development of the Alcohol Use Disorders Identification Test (AUDIT): WHO Collaborative Project on Early Detection of Persons with Harmful Alcohol Consumption-II. Addiction; 88(6):791-804.

Sheron N., Hawkey C., Gilmore I. (2011) Projections of alcohol deaths - a wake-up call. Lancet .

Siegler V, Langford A and Johnson B (2008). Regional differences in male mortality inequalities using the National Statistics Socio-economic Classification, England and Wales, 2001-03. Health Statistics Quarterly 40, 6-26. Available on the Office for National Statistics website at: www.statistics.gov.uk/statbase/product.asp?vlnk $=6725$

White C, Edgar G and Siegler V (2008). Social inequalities in male mortality for selected causes of death by the National Statistics Socio-economic Classification, England and Wales, 2001-03. Health Statistics Quarterly 38, 19-32. Available on the Office for National Statistics website at: www.statistics.gov.uk/statbase/product.asp?vlnk=6725

White C, Glickman M, Johnson B and Corbin T (2007). Social inequalities in adult male mortality by the National Statistics Socio-economic Classification, England and Wales, 2001-03. Health Statistics Quarterly 36, 6-22. Available on the Office for National Statistics website at: www.statistics.gov.uk/statbase/product.asp?vlnk $=6725$

World Health Organization (2011). WHO Global Status Report on Alcohol and Health 2011 Available at: www.who.int/substance abuse/publications/global alcohol report/en/index.html

World Health Organization, Europe (2010). European Status Report on Alcohol and Health 2010. Available at: www.euro.who.int/ data/assets/pdf file/0004/128065/e94533.pdf

World Health Organization (2004). WHO Global Status Report on Alcohol 2004. Available at: www.who.int/substance abuse/publications/global status report 2004 overview.pdf 